\title{
On the X-ray emission of $z \sim 2$ radio galaxies: IC scattering of the CMB and no evidence for fully-formed potential wells
}

\author{
R. A. Overzier ${ }^{1}$, D. E. Harris ${ }^{2}$, C. L. Carilli ${ }^{3}$, L. Pentericci ${ }^{4}$, H. J. A. Röttgering ${ }^{1}$, and G. K. Miley ${ }^{1}$ \\ ${ }^{1}$ Leiden Observatory, University of Leiden, PO Box 9513, 2300 RA Leiden, The Netherlands \\ e-mail: overzier@strw.leidenuniv.nl \\ 2 Smithsonian Astronomical Observatory, Harvard-Smithsonian Center for Astrophysics, 60 Garden Street, Cambridge, \\ MA 02138, USA \\ 3 National Radio Astronomy Observatory, New Mexico Array Operations Center (VLA, VLBA), PO Box O, \\ 1003 Lopezville Road, Socorro, NM 87801, USA \\ ${ }^{4}$ Dipartimento di Fisica, Università degli Studi Roma Tre, Rome, Italy
}

Received 13 July 2004 / Accepted 30 November 2004

\begin{abstract}
We present the results of $20 \mathrm{ks}$ Chandra observations for each of 5 radio galaxies in the redshift range $2.0<z<2.6$. The goals were to (i) study the nature of their non-thermal X-ray emission; (ii) investigate the presence and amount of hot gas; and (iii) look for active galactic nuclei (AGN) overdensities in fields around high redshift radio galaxies. For 4 of the 5 targets we detect unresolved X-ray components coincident with the radio nuclei. From spectral analysis of one of the cores and comparison to the empirical radio to X-ray luminosity ratio $\left(L_{R} / L_{\mathrm{X}}\right)$ correlation for AGN, we find that the cores are underluminous in the $\mathrm{X}$-rays indicating that obscuring material $\left(n(\mathrm{HI}) \sim 10^{22} \mathrm{~cm}^{-2}\right)$ may be surrounding the nuclei.

We detect X-ray emission coincident with the radio hotspots or lobes in 4 of the 5 targets. This extended emission can be explained by the Inverse-Compton (IC) scattering of photons that make up the cosmic microwave background (CMB). The magnetic field strengths of $\sim 100-200 \mu \mathrm{G}$ that we derive agree with the equipartition magnetic field strengths. The relative ease with which the lobe X-ray emission is detected is a consequence of the $(1+z)^{4}$ increase in the energy density of the CMB. For one of the lobes, the X-ray emission could also be produced by a reservoir of hot, shocked gas. An HST image of the region around this radio component shows bright optical emission reminiscent of a bow-shock.

By co-adding the 5 fields we created a deep, $100 \mathrm{ks}$ exposure to search for diffuse X-ray emission from thermal intra-cluster gas. We detect no diffuse emission and derive upper limits of $\sim 10^{44} \mathrm{erg} \mathrm{s}^{-1}$, thereby ruling out a virialized structure of cluster-size scale at $z \sim 2$.

The average number of soft X-ray sources in the field surrounding the radio sources is consistent with the number density of AGN in the Chandra Deep Fields, with only one of the fields showing a marginally statistically significant factor 2 excess of sources with $f_{0.5-2 \mathrm{keV}}>3 \times 10^{-15} \mathrm{erg} \mathrm{s}^{-1} \mathrm{~cm}^{-2}$. Analysis of the angular distribution of the field sources shows no evidence for large-scale structure associated with the radio galaxies, as was observed in the case of PKS 1138-262 by Pentericci et al. (2002).
\end{abstract}

Key words. galaxies: high-redshift - galaxies: active - X-rays: galaxies: clusters - X-rays: general

\section{Introduction}

Radio galaxies can be used to trace the formation and evolution of the most massive galaxies known at high redshift. They usually have continuum morphologies suggestive of the merging of $L_{*}$ systems (e.g. Pentericci et al. 1999, 2001), and are surrounded by large reservoirs of line emitting gas (e.g. van Ojik et al. 1997) that are comparable in size to $\mathrm{cD}$ galaxy envelopes. High redshift radio galaxy (HzRG) fields targeted by broad and narrow band imaging and spectroscopy have been found to locate "protoclusters". They have large excesses of Ly $\alpha$ and/or $\mathrm{H} \alpha$ emitters, Lyman break galaxies and extremely red objects (e.g. Pentericci et al. 2000a; Kurk et al. 2000; Kurk 2003; Venemans et al. 2002; Miley et al. 2004). Thus, HzRGs act as beacons to the progenitors of present-day galaxy clusters (see also e.g. Windhorst et al. 1998; Ivison et al. 2000; Brand et al. 2003; Smail et al. 2003; Stevens et al. 2003). Finding these distant (i.e. $z \gtrsim 2$ ) protoclusters is important for constraining models of structure formation and cosmology.

Radio continuum polarimetric observations of HzRGs have revealed large rotation measures $(R M)$ and large $R M$ gradients. At $z \gtrsim 2$ the typical $R M$ observed is several hundreds $\mathrm{rad} \mathrm{m}^{-2}$, but extreme values of $>1000 \mathrm{rad} \mathrm{m}^{-2}$ are not uncommon (Carilli et al. 1997; Athreya et al. 1998; Pentericci et al. 2000b). It is believed that $R M$ arises from "Faraday screens" in our galaxy (i.e. the interstellar medium, ISM), in intervening galaxies and clusters, or near the source itself. At low redshifts, 
most radio galaxies have relatively small $R M$ values that can be attributed to the local ISM (Simard-Normandin \& Kronberg 1980; Leahy 1987, and references therein). However, $R M$ values observed towards HzRGs are significantly larger, and are believed to be due to Faraday screening of extra-galactic origin somewhere along the line of sight. Athreya et al. (1998) show that the chance superpositions of distant radio sources and intervening systems such as foreground Abell clusters and damped Ly $\alpha$ absorbers are too small to explain the fraction of high $R M$ s observed. Likewise, the expected $R M$ s from such intervening systems are too small to account for the high values seen towards distant radio sources. Hence, the large $R M$ values may occur in a medium close to the source, although it is unclear whether they are caused by extended cluster-sized media or smaller gas distributions on scales comparable to the radio sources (e.g. Carilli et al. 2002). Some radio galaxies at low redshift have extremely large values of $R M$ that are caused by Faraday rotation in so-called "cooling flow" cluster atmospheres (e.g. 3C 295; Perley \& Taylor 1991, see Carilli \& Taylor 2002, for a review on cluster magnetic fields). There is evidence that the strength of cooling flows is correlated with $R M$, indicating that $R M$ is an indicator of the fields in the intracluster medium rather than the radio cocoon (Taylor et al. 1994; Eilek \& Owen 2002; Ensslin et al. 2003, but see Rudnick \& Blundell 2003).

X-ray observations are also a powerful tool to find deep, gravitational potential wells in the early Universe. Diffuse X-ray emission caused by thermal bremsstrahlung from a hot ICM, is evidence for a bound system in which the gas is in dynamical equilibrium with the galaxies and the cold dark matter. Deep X-ray surveys have uncovered significant numbers of rich galaxy clusters out to $z \sim 1.2$ (e.g. Rosati et al. 1999; Stanford et al. 2001; Rosati et al. 2004). Remarkably, the diffuse ICM in these distant objects traces the galaxy distribution, and their surface brightness and temperature profiles are similar to those of lower redshift clusters, indicating that clusters formed very early in the history of the Universe. The study of thermal emission from HzRGs is important because of their suggested linkage to cluster formation. However, if an ICM is present in such structures at $z>1.5$, the extreme cosmological surface brightness dimming makes their detection very difficult.

So far only a handful of distant radio sources has been studied with Chandra. Fabian et al. (2003) performed a very deep (200 ks) study of the radio galaxy 3C 294 at $z=1.79$. They observed a $100 \mathrm{kpc}$ region of diffuse emission bounded by sharp edges. Although some thermal component from the ICM could not be ruled out, most of the emission was ascribed to Inverse Compton (IC) scattering of the cosmic microwave background (CMB) by an older population of electrons tracing out an hourglass-shaped region around the radio source. Belsole et al. (2004) found weak cluster luminosities and IC scattering among a sample of three bright HzRGs. Carilli et al. (2002) found diffuse X-ray emission around the radio galaxy PKS $1138-262$ at $z=2.16$, which is believed to be the forming, massive galaxy at the center of a protocluster (Pentericci et al. 2000a; Kurk et al. 2000; Kurk 2003). However, the extended emission is seen only along the radio axis, and is therefore believed to be associated with shocked material inside the radio source. The upper limit that Carilli et al. (2002) derive for the X-ray luminosity of the ICM ( 40\% of the luminosity of the Cygnus A cluster, see e.g. Smith et al. 2002) is not unexpectedly low because there has not been enough time since the big bang for these structures at $z \sim 2$ to form a sufficiently deep potential well. Scharf et al. (2003) detected extended X-ray emission around $4 \mathrm{C} 41.17$ at $z=3.8$ roughly following the radio morphology. They conclude that the X-ray emission arises from "Inverse-Compton scattering of far-infrared photons from a relativistic electron population probably associated with past and current activity from the central object", in addition to a lesser contribution from the upscattering of CMB photons.

It is clear that the nature of the X-ray emission mechanisms operating in radio galaxies is diverse. Better sample statistics are required to enlarge our current understanding of e.g. the dominant X-ray emission mechanisms, the role of magnetic fields on galaxy and cluster-size scales, the origin of the hot gas observed in X-ray luminous clusters, and large-scale structure associated with HzRGs. Here we present observations with the Chandra X-ray observatory of five additional radio galaxies at $z \sim 2$. The sources were selected from a compendium of $z>2$ HzRGs, focusing on the lower end of the redshift distribution in order to minimize surface brightness dimming effects, while still being at high enough redshift to allow for cosmological evolution effects in source properties. Sources were further selected on the presence of one or more of the various characteristics: distorted radio morphology, large rotation measure $\left(\geq 1000 \mathrm{rad} \mathrm{m}^{-2}\right)$, evidence for interaction between Ly $\alpha$ and radio structure, and extended Ly $\alpha$ absorption features.

Throughout this paper we assume $H_{0}=70 \mathrm{~km} \mathrm{~s}^{-1}$, $\Omega_{\mathrm{M}}=0.3$, and $\Omega_{\Lambda}=0.7$. The resulting scale factors range from 8.0 to $8.4 \mathrm{kpc} \operatorname{arcsec}^{-1}$ from $z=2.6$ to $z=2$. We will use power-law spectra defined as $S_{v} \propto v^{-\alpha}$, where $\alpha$ is the spectral index.

\section{Observations}

\subsection{Observations and data reduction}

Each of the 5 sources was observed for $20 \mathrm{ks}$ with the backilluminated ACIS-S3 chip on Chandra, using the standard $3.2 \mathrm{~s}$ readout timed exposure mode and the faint telemetry format. Sources were positioned at approximately $20^{\prime \prime}$ away from the aimpoint. The data were processed and analysed in June 2003 using the Chandra data analysis package CIAO $2.3^{1}$ together with CALDB 2.22. We applied the "ACISABS" script that corrects the Chandra auxiliary response files for a continuous degredation in the ACIS quantum efficiency. We did not apply the "tgain" correction that has become standard practice only in the most recent release of CIAO to correct for the drift in the effective detector gain due to an increase in the charge transfer inefficiency over time. However, for ACIS-S3 the resulting effect on the measured photon energies was of the order of only $\sim 0.3 \%$ near the end of 2002 when the data were taken.

A log of the observations is given in Table 1. 0156-252 was observed on 2 separate dates, because the initial observation

\footnotetext{
${ }^{1}$ http://cxc.harvard.edu/ciao/
} 
was interrupted after $11.5 \mathrm{ks}$ by a high radiation shutdown of the system due to an excess of solar wind particles. To ease the analysis of these observations, the two exposures were merged into a single event file by "reprojecting" the events. A lightcurve analysis of the other sources indicates no further periods of significant fluctuations in the count rates of source and large, source-free background regions.

Radio images at 5 and $8 \mathrm{GHz}$ where obtained by Carilli et al. (1997) and Pentericci et al. (2000a) using the VLA in A configuration. The noise is $50 \mu \mathrm{Jy} /$ beam at $5 \mathrm{Ghz}$ and $25 \mu \mathrm{Jy} / \mathrm{beam}$ at $8 \mathrm{GHz}$. The resolution of the observations is $0.43^{\prime \prime}$ for the $5 \mathrm{GHz}$ maps and $0.23^{\prime \prime}$ for the $8 \mathrm{GHz}$ maps. Analysis of the radio data was performed using the Astronomical Image Processing System (AIPS) and the Multichannel Image Reconstruction, Image Analysis and Display (MIRIAD) software.

We will also make use of observations obtained with the Wide-Field Planetary Camera 2 (WFPC2) on HST. 0156-252 and 2048-272 were observed as part of Program 8183 (PI: Miley), and an image of 0406-244 was retrieved from the HST archive (program 8338, PI: Lehnert). 0156-252 was observed for $4800 \mathrm{~s}$ and 2048-272 for $7200 \mathrm{~s}$, both through filter $F 555 \mathrm{~W}$. This filter has a central wavelength of $5443 \AA$. 0406-244 was observed for $2000 \mathrm{~s}$ through filter $F 675 \mathrm{~W}$ which has a central wavelength of $6718 \AA$. Measurements from these images were made using the conversion from counts to flux density as given in the PHOTFLAM header keyword of each image. To get to the flux density $S_{v}$ $\left(\mathrm{erg} \mathrm{s}^{-1} \mathrm{~cm}^{-2} \mathrm{~Hz}^{-1}\right)$ we calculate $S_{v}=\left(\lambda^{2} / c\right) \times\left(\right.$ counts $\left./ t_{\mathrm{exp}}\right) \times$ PHOTFLAM (see Pentericci et al. 1999, for a description of procedures).

\subsection{Image registration}

To recover the inherent resolution of the Chandra mirror/detector system, we removed the pixel randomization added in the pipeline processing and regridded our maps to $1 / 10$ native ACIS pixel size. After applying a suitable smoothing function, we were able to find the position of the X-ray core to an accuracy of better than $0.1^{\prime \prime}$ provided the core had sufficient signal to noise $(S / N)$. We then shifted the images to align the $\mathrm{X}$-ray core with the radio core. The typical shift needed was one half of an original Chandra pixel in right ascension and/or declination, consistent with the known astrometric accuracy. In the case of 2048-272 no shift was applied, because the nucleus was not detected in either the radio or the X-rays.

\subsection{Analysis}

The Chandra images are shown in Fig. 1, where yellow contours indicate the $4.7 \mathrm{GHz}$ radio maps from Carilli et al. (1997) and Pentericci et al. (2000b). We measured the X-ray counts from the distinct radio components (e.g. cores and lobes) using boxed or circular extraction regions. The counts are background-subtracted by using a large, point source free, annular region centered on the radio source. We restrict our measurements to counts in the energy range of $0.2-6 \mathrm{keV}$ for which the contribution of the background is minimal. We calculate the total net (i.e. background subtracted) counts $N=S-B$, where $S$ and $B$ are the counts in the source region $A_{\mathrm{S}}$ and the background region $A_{\mathrm{B}}$, respectively. We consider a radio component detected in the X-rays when $N$ is greater than its formal error, $\sigma_{N}=\left[\left(\sigma_{\mathrm{S}}\right)^{2}+\left(\sigma_{\mathrm{B}}\right)^{2}\right]^{1 / 2}$, where $\sigma_{\mathrm{S}}=1+(S+0.75)^{1 / 2}$ and $\sigma_{\mathrm{B}}=\left[1+(B+0.75)^{1 / 2}\right]\left(A_{\mathrm{S}} / A_{\mathrm{B}}\right)$ from Gehrels (1986). For undetected components we calculate $2 \sigma$ upper limits in the $0.2-6 \mathrm{keV}$ band using the above expression for $\sigma_{N}$. The upper limit on flux is estimated by assuming a power-law spectrum with a spectral index of 0.8 using the online tool PIMMS ${ }^{2}$.

The procedure to convert counts into fluxes is as follows: we create exposure maps for the soft $(0.5-2 \mathrm{keV})$ and hard $(2-6 \mathrm{keV})$ band at nominal energies, and divide the images in each band by the corresponding exposure map to obtain fluxed images. When extracting the flux for a given region, we calculate weight factors defined by the ratio of the average energy of counts in that region to the nominal energy of each exposure map. The total flux in a region is then calculated by summing the weighted fluxes in the two bands. We remark that our method is slightly different compared to the usual procedure of calculating fluxes from exposure maps using a conversion factor that is based on the spectral response within the given band as well as an assumed spectral energy distribution. Our method is preferred because it allows us to measure fluxes, without having to assume a specific spectral shape.

The measurements are given in Table 3. Where indicated, fluxes are corrected for galactic absorption using PIMMS, assuming a power-law spectrum with a spectral index of 0.8 and taking the HI column density in the direction of each source from Dickey \& Lockman (1990) indicated in Table 1. All energy ranges are in the observed frame, unless stated otherwise.

\section{Results}

\subsection{Source description}

We will briefly summarize some of the main characteristics of each source. Details on radio observations, ground-based imaging and spectroscopy, and Hubble Space Telescope (HST) observations can be found in McCarthy et al. (1996), Carilli et al. (1997), Pentericci et al. (1999, 2001), Iwamuro et al. (2003) and references therein. Table 2 summarizes the intrinsic rotation measures, $R M_{\mathrm{int}}=R M_{\mathrm{obs}} \times(1+z)^{2}$.

- 0156-252 at $z=2.09$ has several characteristics reminiscent of the well-studied radio galaxy PKS 1138-262 (Carilli et al. 2002; Pentericci et al. 2000a). Near-infrared continuum shows a host galaxy extended over $\sim 2$ " parallel to the radio axis, and narrow-band Ly $\alpha$ shows emission line gas extended over the entire region encompassed by the northern and southern radio hot spots. The Ly $\alpha$ brightness is at a minimum at the position of the host galaxy and peaks at the location where there is a sharp bend in the (northern) radio jet, probably due to shock-induced ionization of the gas where the radio jet is deflected by denser material. The northern hot spot has an intrinsic $R M$ of $\sim 1000 \mathrm{rad} \mathrm{m}^{-2}$.

\footnotetext{
${ }^{2}$ http://heasarc.gsfc.nasa.gov/Tools/w3pimms.html
} 

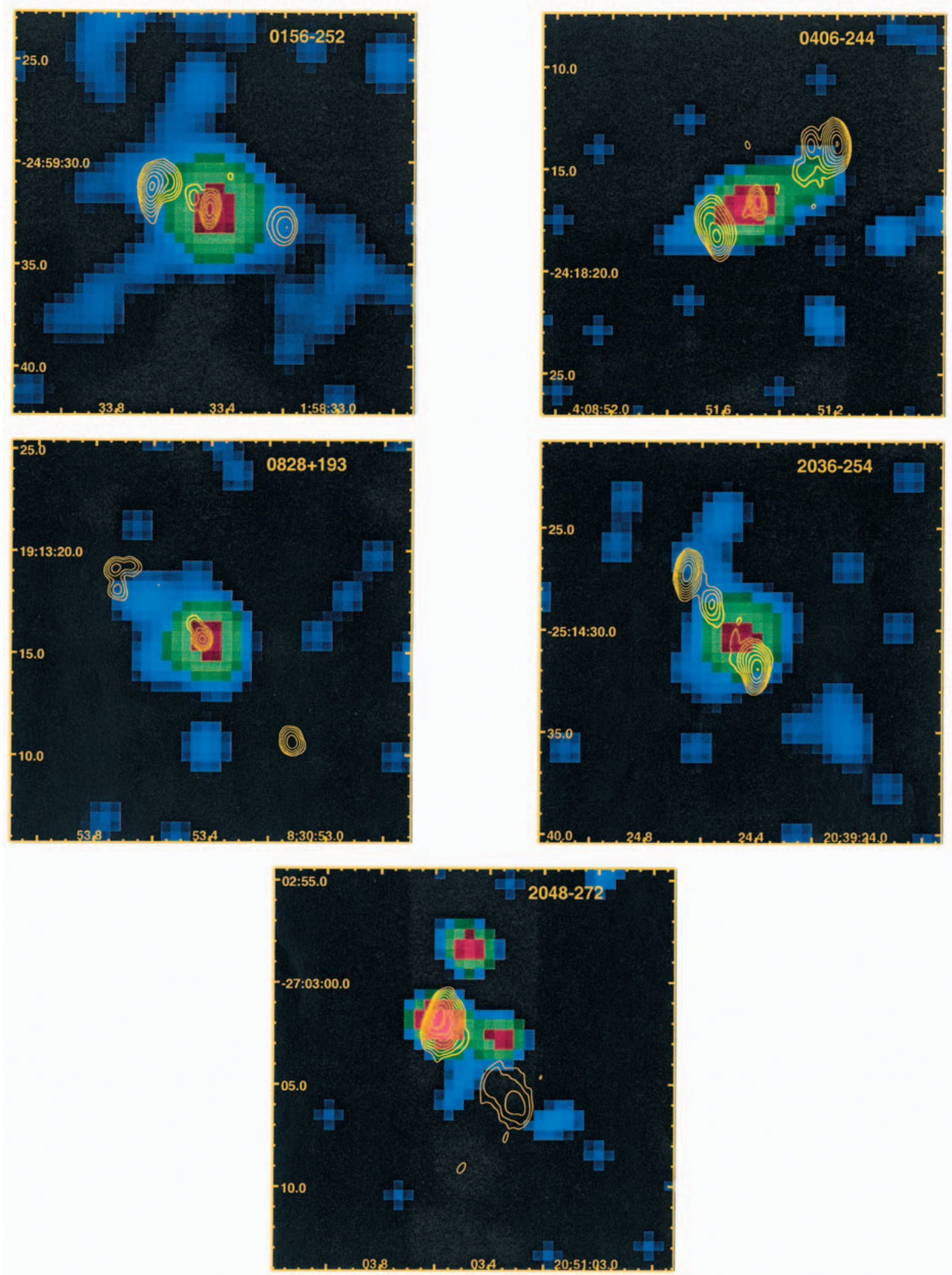

Fig. 1. Chandra images of 0156-252, 0406-244, 0828+193, 2036-254 and 2048-272, showing the 0.2-6 keV X-ray images in colorscales, and the 4.7 GHz VLA radio contours in yellow (from Carilli et al. 1997; Pentericci et al. 2000a). The 20" $\times 20^{\prime \prime}$ Chandra images have been smoothed using a Gaussian kernel of width $2^{\prime \prime}(F W H M)$. Radio contours are $1.0 \times(0.2,0.4,0.8,1.6, \ldots) \mathrm{mJy}$ beam $^{-1}$. 
Table 1. Log of observations. The last column lists the average galactic HI column density in the direction of each source as given by Dickey \& Lockman (1990).

\begin{tabular}{ccccccc}
\hline \hline Source & RA $(\mathrm{J} 2000)$ & Dec $(\mathrm{J} 2000)$ & $z$ & Dates of observation & Exposure time $(\mathrm{s})$ & $n(\mathrm{HI})\left(\times 10^{20} \mathrm{~cm}^{-2}\right)$ \\
\hline $0156-252$ & $01^{\mathrm{h}} 58^{\mathrm{m}} 33.5^{\mathrm{s}}$ & $-24^{\circ} 59^{\prime} 32.26^{\prime \prime}$ & 2.09 & $2002-11-09$ & 11477 & 1.34 \\
& & & & $2002-12-20$ & 8401 & 3.30 \\
$0406-244$ & $04^{\mathrm{h}} 08^{\mathrm{m}} 51.5^{\mathrm{s}}$ & $-24^{\circ} 18^{\prime} 16.8^{\prime \prime}$ & 2.44 & $2002-12-07$ & 20179 & 3.72 \\
$0828+193$ & $08^{\mathrm{h}} 30^{\mathrm{m}} 53.4^{\mathrm{s}}$ & $+19^{\circ} 13^{\prime} 15.7^{\prime \prime}$ & 2.57 & $2002-11-05$ & 19212 & 4.92 \\
$2036-254$ & $20^{\mathrm{h}} 39^{\mathrm{m}} 24.5^{\mathrm{s}}$ & $-25^{\circ} 14^{\prime} 30.4^{\prime \prime}$ & 2.00 & $2002-09-01$ & 19660 & 6.29 \\
$2048-272$ & $20^{\mathrm{h}} 51^{\mathrm{m}} 03.6^{\mathrm{s}}$ & $-27^{\circ} 03^{\prime} 02.1^{\prime \prime}$ & 2.06 & $2002-11-11$ & 19555 & \\
\hline
\end{tabular}

Table 2. Rotation measures, $R M_{\mathrm{int}}=R M_{\mathrm{obs}} \times(1+z)^{2}$, for the Northern (N.) and Southern (S.) lobes of each source taken from the literature.

\begin{tabular}{|c|c|c|c|}
\hline \multirow[t]{2}{*}{ Source } & \multicolumn{2}{|c|}{$R M_{\text {int }}\left(\mathrm{rad} \mathrm{m}^{-2}\right)$} & \multirow[t]{2}{*}{ Ref. } \\
\hline & N. lobe & S. lobe & \\
\hline $0156-252$ & $1528 \pm 296$ & - & 1 \\
\hline 0406-244 & $880 \pm 19$ & $-705 \pm 30$ & 1 \\
\hline $0828+193$ & - & - & 2 \\
\hline $2036-254$ & $3 \pm 227$ & $-3321 \pm 429$ & 1 \\
\hline $2048-272$ & 590 & - & 3 \\
\hline
\end{tabular}

1 Athreya et al. (1998).

2 Carilli et al. (1997).

3 Pentericci et al. (2000).

- 0406-244 at $z=2.44$ consists of two main optical/infrared continuum features aligned with the radio axis (see Rush et al. 1997 for a detailed study). The continuum components lie embedded in a large Ly $\alpha$ halo with a spatial extent of $\sim 8^{\prime \prime}$ along the radio axis. Both radio lobes have an intrinsic $R M$ of $\sim 1000 \mathrm{rad} \mathrm{m}^{-2}$ with opposite signs.

- $0828+193$ at $z=2.57$ is the largest radio source in our sample $(\sim 100 \mathrm{kpc})$. It has a jet-like feature north of the radio core. The northern lobe contains several hot spots with a peculiar 90 degree bend. The southern radio hotspot is unresolved. Pentericci et al. (1999) have identified the radio core with the brightest optical component of several clumps in a $\sim 2^{\prime \prime}$ region seen by HST.

No $R M$ is given for this source in Table 2, since the fractional polarization at $8.2 \mathrm{GHz}$ for both lobes was below the $4 \sigma$ sensitivity limit of the observations (Carilli et al. 1997).

- 2036-254 at $z=2.00$ is the only source in our sample for which no optical/near-infrared data are available. The radio core is most likely the faint, compact component close to the southern lobe. There is a pair of hot spots parallel to the radio axis in the north. The southern lobe has a $R M_{\mathrm{int}}$ of $\sim 3500 \mathrm{rad} \mathrm{m}^{-2}$.

- 2048-272 at $z=2.06$ is among the $\sim 30 \%$ of sources in the sample of Carilli et al. (1997) for which the core is undetected. However, a bright near-infrared object seen in between the lobes is presumed to be the galaxy hosting the radio source. The northern hot spot has a $R M_{\text {int }}$ of $\sim 590 \mathrm{rad} \mathrm{m}^{-2}$.

\subsection{X-ray cores}

We have detected X-ray cores in 4 of the 5 targets. No core was detected in the case of 2048-272, and we note that the core was also not detected in the radio maps indicating that nuclear activity may have ceased. The core $\mathrm{X}$-ray luminosities are in the range $L_{\mathrm{X}[0.5-6 \mathrm{keV}]}=1.3 \times 10^{44}-2.3 \times 10^{45} \mathrm{erg} \mathrm{s}^{-1}$. 0156-252 has $>4 \times$ the luminosity of the other cores. We place an upper limit on the core luminosity of 2048-272 of $7 \times 10^{43} \mathrm{erg} \mathrm{s}^{-1}$. Hardcastle \& Worrall (1999) and Brinkmann et al. (2000) presented a correlation between the X-ray and radio luminosity of radio-loud quasars from the cross-correlation of ROSAT and radio sources. It has been suggested that this correlation implies a physical relationship between X-ray and radio cores, which can be explained by models in which e.g. the X-ray emission originates from the base of the jet (Hardcastle \& Worrall 1999). In Fig. 2 we show objects from the Brinkmann et al. (2000) sample classified as narrow-line AGN, as well as the best-fit correlation for their subsample of radio-loud quasars, $\log L_{\mathrm{X}}=11.22+0.483 \times \log L_{r}$. We have also indicated several other $z>2$ radio galaxies (1138-262 at $z=2.16, \mathrm{~B} 0902+343$ at $z=3.395,4 \mathrm{C} 41.17$ at $z=3.798)$ and the local source Cygnus A for comparison (see Carilli et al. 2002; Scharf et al. 2003; Fabian et al. 2002, and references therein).

As shown in Fig. 2, three out of four sources in our sample agree with the $L_{r} / L_{\mathrm{X}}$ relation if we use the observed 2-6 keV (rest-frame 6-18 keV) flux with a power-law spectrum $(\alpha=0.8)$ to calculate the luminosity at rest-frame $2 \mathrm{keV}$ (observed $0.7 \mathrm{keV}$ ). This result is virtually independent of the amount of intrinsic absorption, since it is of negligible effect at rest-frame $6-18 \mathrm{keV}$. Calculating the $2 \mathrm{keV}$ luminosity from the $0.5-2 \mathrm{keV}$ observed flux (rest-frame $1.5-6 \mathrm{keV}$ ) yields values that are significantly lower, but for 0156-252, 0828+193 and 2036-254 this can be explained by an intrinsic absorption column density of $n(\mathrm{HI}) \sim 10^{23} \mathrm{~cm}^{-2}$. Interestingly, the core of 0406-244 seems highly underluminous in Fig. 2, a discrepancy that cannot be explained away by invoking a large amount of absorption. However, this may be a $S / N$ issue: we have detected only 2 photons in the hard 2-6 keV band, compared to 62,10 and 15 for the other 3 sources. If we instead calculate the $2 \mathrm{keV}$ luminosity from the $0.5-2 \mathrm{keV}$ observed flux (8 photons detected) with $n(\mathrm{HI})=10^{23} \mathrm{~cm}^{-2}$ we find $\log \left(L_{2 \mathrm{keV}}\right) \approx 26.4$ compared to $\sim 25.9$ for the earlier method, and note that its deviation from the $L_{r} / L_{\mathrm{X}}$ relation is now comparable to that of $4 \mathrm{C} 41.17$. 
Table 3. X-ray measurements.

\begin{tabular}{|c|c|c|c|c|c|c|c|}
\hline Source & $\begin{array}{l}\theta^{e} \\
\left({ }^{\prime \prime}\right)\end{array}$ & $\begin{array}{c}\text { Counts }^{a} \\
(0.2-6 \mathrm{keV})\end{array}$ & $\begin{array}{c}\text { Soft }^{b} \\
(0.5-2 \mathrm{keV})\end{array}$ & $\begin{array}{c}\text { Hard }^{b} \\
(2-6 \mathrm{keV})\end{array}$ & $\begin{array}{c}f_{0.5-2 \mathrm{keV}}^{c} \\
\operatorname{erg~s}^{-1} \mathrm{~cm}^{-2}\end{array}$ & $\begin{array}{c}f_{2-6 \mathrm{keV}}^{c} \\
\operatorname{erg~s}^{-1} \mathrm{~cm}^{-2}\end{array}$ & $\begin{array}{c}\log \left(L_{0.5-6 \mathrm{keV}}\right) \\
\operatorname{erg~s}^{-1}\end{array}$ \\
\hline \multicolumn{8}{|c|}{$0156-252$} \\
\hline Total & 4.2 & $275 \pm 18$ & $200 \pm 14.2$ & $69 \pm 8.3$ & $(3.1 \pm 0.2) \times 10^{-14}$ & $(4.9 \pm 0.6) \times 10^{-14}$ & 45.42 \\
\hline Core & 1.1 & $249 \pm 17$ & $181 \pm 13.5$ & $62 \pm 7.9$ & $(2.8 \pm 0.2) \times 10^{-14}$ & $(4.4 \pm 0.6) \times 10^{-14}$ & 45.37 \\
\hline $\mathrm{NE}$ & 1.6 & $7 \pm 4$ & $4.9 \pm 2.2$ & $1.0 \pm 1.0$ & $(7.4 \pm 3.4) \times 10^{-16}$ & $(3.9 \pm 4.7) \times 10^{-16}$ & 43.56 \\
\hline SW & 1.2 & $4 \pm 3$ & $1.9 \pm 1.4$ & $1.9 \pm 1.4$ & $(4.6 \pm 3.4) \times 10^{-16}$ & $(1.6 \pm 1.2) \times 10^{-15}$ & 43.82 \\
\hline \multicolumn{8}{|c|}{$0406-244$} \\
\hline Total & 4.8 & $21 \pm 6$ & $18 \pm 4.4$ & $0.5 \pm 1.4$ & $(2.9 \pm 0.7) \times 10^{-15}$ & $(3.6 \pm 9.3) \times 10^{-16}$ & 44.19 \\
\hline Core & 0.9 & $10 \pm 4$ & $8.0 \pm 2.8$ & $2.0 \pm 1.4$ & $(1.4 \pm 0.5) \times 10^{-15}$ & $(1.3 \pm 0.9) \times 10^{-15}$ & 44.11 \\
\hline $\mathrm{SE}$ & 1.8 & $9 \pm 4$ & $6.9 \pm 2.6$ & $1.0 \pm 1.0$ & $(1.0 \pm 0.4) \times 10^{-15}$ & $(5.2 \pm 6.5) \times 10^{-16}$ & 43.88 \\
\hline NW & 2.0 & $\ldots{ }^{d}$ & \multicolumn{2}{|c|}{$\ldots$} & \multicolumn{2}{|c|}{$<2.4 \times 10^{-15}$} & $<44.05$ \\
\hline \multicolumn{8}{|c|}{$0828+193$} \\
\hline Total & 7.0 & $32 \pm 7$ & $17 \pm 4.4$ & $13.3 \pm 4.0$ & $(3.2 \pm 0.8) \times 10^{-15}$ & $(1.2 \pm 0.4) \times 10^{-14}$ & 44.91 \\
\hline Core & 1.0 & $23 \pm 6$ & $12 \pm 3.5$ & $9.9 \pm 3.2$ & $(2.1 \pm 0.6) \times 10^{-15}$ & $(8.7 \pm 2.8) \times 10^{-15}$ & 44.78 \\
\hline $\mathrm{NE}$ & 1.4 & $\ldots{ }^{d}$ & \multicolumn{2}{|c|}{$\ldots$} & \multicolumn{2}{|c|}{$<1.9 \times 10^{-15}$} & $<44.00$ \\
\hline SW & 1.0 & $\ldots^{d}$ & \multicolumn{2}{|c|}{$\ldots$} & \multicolumn{2}{|c|}{$<1.6 \times 10^{-15}$} & $<43.93$ \\
\hline \multicolumn{8}{|c|}{$2036-254$} \\
\hline Total & 4.1 & $35 \pm 7$ & $14 \pm 3.9$ & $18.5 \pm 4.4$ & $(2.6 \pm 0.7) \times 10^{-15}$ & $(1.2 \pm 0.3) \times 10^{-14}$ & 44.65 \\
\hline Core & 0.6 & $22 \pm 6$ & $7.0 \pm 2.6$ & $15.0 \pm 3.9$ & $(1.4 \pm 0.5) \times 10^{-15}$ & $(9.6 \pm 2.5) \times 10^{-15}$ & 44.51 \\
\hline $\mathrm{NE}$ & 1.3 & $\ldots{ }^{d}$ & \multicolumn{2}{|c|}{$\ldots$} & \multicolumn{2}{|c|}{$<2.0 \times 10^{-15}$} & $<43.76$ \\
\hline SW & 1.4 & $6 \pm 4$ & $5.9 \pm 2.5$ & $0.0 \pm 0.0$ & $(1.0 \pm 0.4) \times 10^{-15}$ & $\ldots$ & 43.47 \\
\hline \multicolumn{8}{|c|}{$2048-272$} \\
\hline Total & 4.3 & $8 \pm 4$ & $4.1 \pm 2.2$ & $4.0 \pm 2.2$ & $(6.0 \pm 3.3) \times 10^{-16}$ & $(2.9 \pm 1.6) \times 10^{-15}$ & 44.05 \\
\hline Core & 0.8 & $\ldots{ }^{d}$ & \multicolumn{2}{|c|}{$\ldots$} & \multicolumn{2}{|c|}{$<2.1 \times 10^{-15}$} & $<43.82$ \\
\hline $\mathrm{NE}$ & 1.7 & $6 \pm 4$ & $2.8 \pm 1.7$ & $1.8 \pm 1.4$ & $(4.5 \pm 2.8) \times 10^{-16}$ & $(1.2 \pm 1.0) \times 10^{-15}$ & 43.73 \\
\hline SW & 1.5 & $\ldots{ }^{d}$ & \multicolumn{2}{|c|}{$\ldots$} & \multicolumn{2}{|c|}{$<1.7 \times 10^{-15}$} & $<43.72$ \\
\hline
\end{tabular}

${ }^{a}$ Errors are calculated using Gehrels (1986) in the low-count regime.

${ }^{b}$ Errors are $\sqrt{\text { counts. }}$

${ }^{c}$ Observed fluxes (i.e. not corrected for galactic absorption).

${ }^{d}$ Undetected. We give a $2 \sigma$ upper limit for the total flux at $0.2-6 \mathrm{keV}$.

${ }^{e}$ Circular extraction radius.

0156-252 is the only source in our sample for which the core is detected with sufficient $S / N$ to carry out a (crude) spectral analysis. The X-ray spectrum is shown in Fig. 3. Assuming a power-law spectrum with absorption at the redshift of the source, we find $\alpha_{\mathrm{X}}=0.8 \pm 0.2$ and $n(\mathrm{HI})=(1.6 \pm 0.7) \times$ $10^{22} \mathrm{~cm}^{-2}$. The fit has a reduced $\chi^{2}$ of 0.34 , and is indicated in Fig. 3. The error bars for the fit parameters correspond to a change of 1 in the reduced $\chi^{2}$. Repeating the fit using using the Cash statistic valid for the low counts regime we find $n(\mathrm{HI})=(1.5 \pm 0.8) \times 10^{22} \mathrm{~cm}^{-2}$ with $\alpha$ unchanged. The galactic HI column density towards this source is $1.34 \times 10^{20} \mathrm{~cm}^{-2}$ (see Table 1). For completeness, we note that the spectrum can be fitted by a Mewe-Kaastra-Liedahl (Mekal) thermal plasma model with $k T=23 \pm 8 \mathrm{keV}$ (reduced $\chi^{2}$ of 0.50 ).

The signal-to-noise of the 3 other cores is insufficient to make spectra. However, if we assume that they are a generic class we might estimate the amount of intrinsic absorption by stacking the data. The hardness ratio $H R \equiv(H-S) /(H+S)$ (where $H$ and $S$ are the number counts observed in the hard and soft bands) is $0.0 \pm 0.2$, consistent with a type II AGN at $z \sim 2$ with an obscured $\left(n(\mathrm{HI}) \approx 10^{23} \mathrm{~cm}^{-2}\right)$ power-law spectrum with a photon index of $\Gamma=1.8$ (Tozzi et al. 2001).

\subsection{X-ray emission from lobes/hotspots}

We obtained positive detections of X-ray components coincident with the northeastern (NE) and southwestern (SW) radio lobes of 0156-252, the southestern (SE) lobe of 0406-244, the SW lobe of 2036-254, and the NE lobe of 2048-272. Below we will evaluate the likely mechanisms for these detections. Given the generally low signal to noise of all features we detected, we will focus on the X-ray emission that is identified with the radio lobes ${ }^{3}$.

\footnotetext{
${ }^{3}$ Our radio data are inadequate to confidently identify radio features as lobes or hotspots. We adopt the term lobes to mean any radio emission not associated with the core.
} 
Table 4. Synchrotron models.

\begin{tabular}{|c|c|c|c|c|c|c|c|c|}
\hline Component & $\begin{array}{c}S_{4.7 \mathrm{GHz}}^{a} \\
(\mathrm{mJy})\end{array}$ & $\begin{array}{c}S_{8.2 \mathrm{GHz}}^{a} \\
(\mathrm{mJy})\end{array}$ & $\alpha_{4.7}^{8.2 b}$ & $\begin{array}{c}S_{\mathrm{X}, \text { model }}^{c} \\
(\mathrm{Jy})\end{array}$ & $\begin{array}{c}S_{\mathrm{X}, \mathrm{obs}}^{d} \\
(\mathrm{Jy})\end{array}$ & $\alpha_{4.7}^{\mathrm{X} e}$ & $\begin{array}{c}S_{8.2 \mathrm{GHz} \text {,model }}^{f} \\
(\mathrm{mJy})\end{array}$ & $\begin{array}{c}S_{5000 \AA \text { 丹̊model }}^{g} \\
(\mathrm{Jy})\end{array}$ \\
\hline $0156-252 \mathrm{NE}$ & 89 & 47 & 1.15 & $5.0 \times 10^{-11}$ & $8.5 \times 10^{-11}$ & 1.13 & 48 & $1.6 \times 10^{-7}$ \\
\hline SW & 7 & 3.5 & 1.25 & $6.4 \times 10^{-13}$ & $1.5 \times 10^{-10}$ & 0.96 & 4.1 & $9.2 \times 10^{-8}$ \\
\hline 0406-244 SE & 31 & 11 & 1.86 & $4.8 \times 10^{-17}$ & $1.2 \times 10^{-10}$ & 1.05 & 17 & $1.4 \times 10^{-7}$ \\
\hline NW & 72 & 32 & 1.46 & $1.5 \times 10^{-13}$ & $<1.8 \times 10^{-10}$ & $>1.07$ & $<40$ & $<2.4 \times 10^{-7}$ \\
\hline $0828+193 \mathrm{NE}$ & 7 & 2.0 & 2.25 & $1.0 \times 10^{-20}$ & $<1.4 \times 10^{-10}$ & $>0.96$ & $<4.1$ & $<8.7 \times 10^{-8}$ \\
\hline SW & 9 & 3.6 & 1.65 & $6.0 \times 10^{-16}$ & $<1.2 \times 10^{-10}$ & $>0.98$ & $<5.2$ & $<8.6 \times 10^{-8}$ \\
\hline $2036-254 \mathrm{NE}$ & 51 & 29 & 1.01 & $3.7 \times 10^{-10}$ & $<1.5 \times 10^{-10}$ & $>1.06$ & $<28$ & $<1.9 \times 10^{-7}$ \\
\hline SW & 37 & 15 & 1.62 & $4.2 \times 10^{-15}$ & $7.7 \times 10^{-11}$ & 1.08 & 20 & $1.1 \times 10^{-7}$ \\
\hline $2048-272 \mathrm{NE}$ & 83 & 34 & 1.60 & $1.4 \times 10^{-14}$ & $1.3 \times 10^{-10}$ & 1.10 & 45 & $2.0 \times 10^{-7}$ \\
\hline SW & 6 & 1.1 & 3.05 (2.2 used) & $2.1 \times 10^{-20}$ & $<1.3 \times 10^{-10}$ & $>0.96$ & $<3.5$ & $<7.7 \times 10^{-8}$ \\
\hline
\end{tabular}

${ }^{a}$ Integrated radio flux density at 4.7 and $8.2 \mathrm{GHz}$. The typical error is assumed to be $\sim 5 \%$.

${ }^{b}$ Radio spectral index. The typical error is assumed to be $\sim 0.2$.

${ }^{c}$ Predicted flux density at $2 \mathrm{keV}$ for a radio synchrotron model.

${ }^{d}$ Observed flux density at $2 \mathrm{keV}$.

${ }^{e} \mathrm{X}$-ray to $(4.7 \mathrm{GHz})$ radio spectral index.

${ }^{f}$ Predicted flux density at $8.2 \mathrm{GHz}$.

${ }^{g}$ Predicted flux density at $5000 \AA$ A.

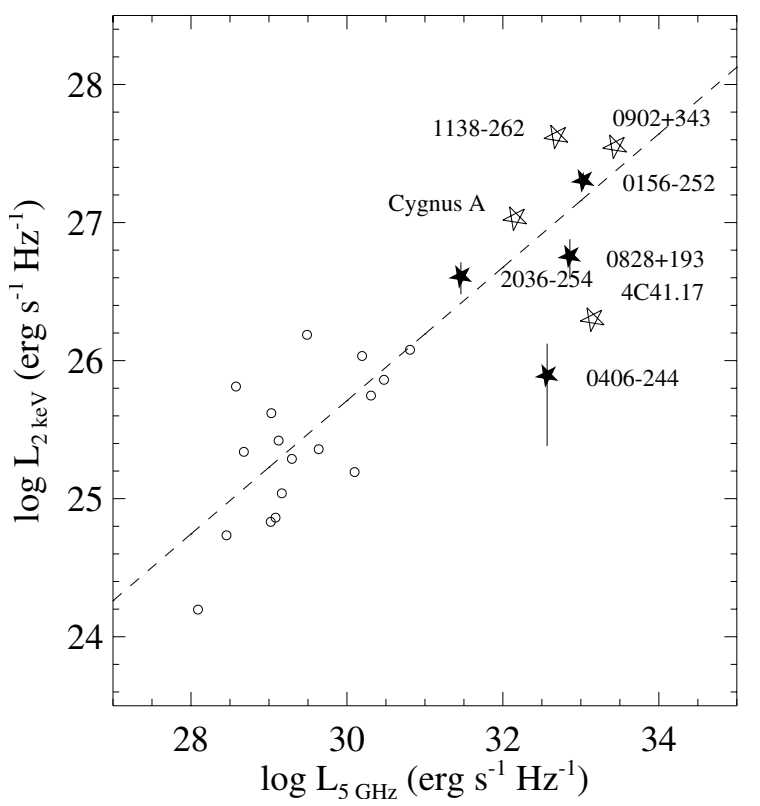

Fig. 2. Monochromatic X-ray luminosity versus radio luminosity. The four detected cores from our sample are indicated by filled stars. Their X-ray luminosities were corrected for galactic absorption only. Narrow line AGN from the FIRST/ROSAT sample of Brinkmann et al. (2000) are indicated by circles. The dashed line indicates the correlation for radio-loud quasars of Brinkmann et al. (2000). Open stars indicate several low (Cygnus A at $z=0.06$ ) and high redshift (1138-262 at $z=2.16, \mathrm{~B} 0902+343$ at $z=3.395,4 \mathrm{C} 41.17$ at $z=3.798)$ radio galaxies from the literature (see Carilli et al. 2002; Scharf et al. 2003; Fabian et al. 2002, and references therein). The X-ray luminosities of these sources were all corrected for intrinsic absorption.

- Synchrotron radiation

In Fig. 4 we plot spectra for the lobes that have a detection in the X-ray. The spectra were constructed by (i) extrapolating the
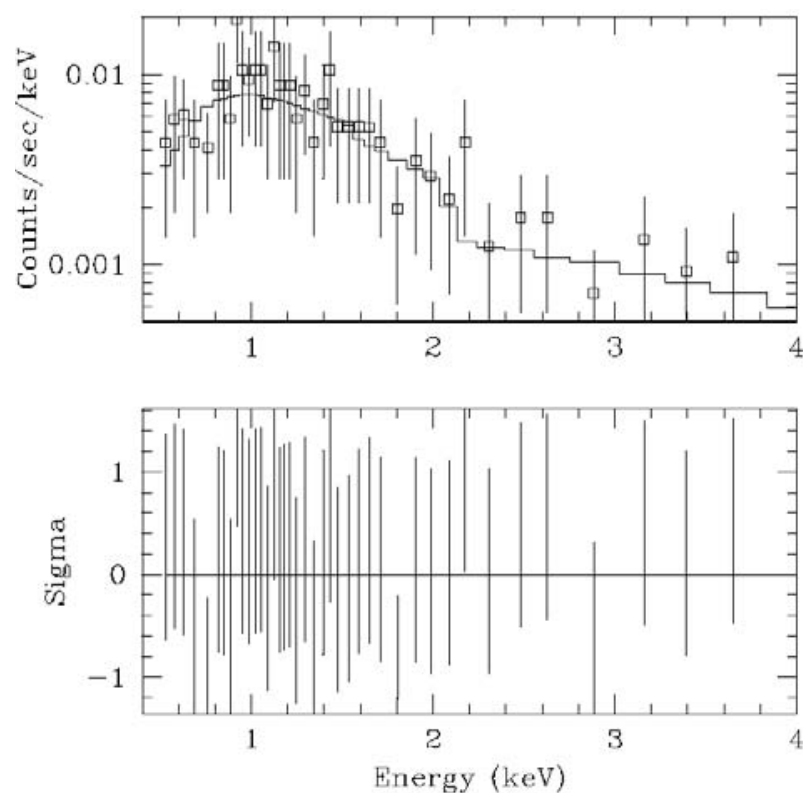

Fig. 3. Spectrum of the core of 0156-252, fitted with a power-law spectrum modified by absorption at the source.

radio flux densities to $\mathrm{X}$-ray frequencies using the radio spectral index $\alpha_{4.7}^{8.2}$; and (ii) using the $4.7 \mathrm{GHz}$ to X-ray spectral index, $\alpha_{4.7}^{\mathrm{X}}$. The results of our analysis are indicated in Table 4. In the first method (indicated by dotted lines in Fig. 4), we can directly compare the observed X-ray flux density to the predicted synchrotron flux density based on the radio spectrum. Except for the NE lobe of 0156-252, the predicted flux densities are several orders of magnitude lower than that observed. This seems to rule out a synchrotron origin for the X-ray emission from these lobes. However, $S_{v}$ is a strong function of the spectral index, since we are extrapolating over $\sim 10^{8}$ decades 


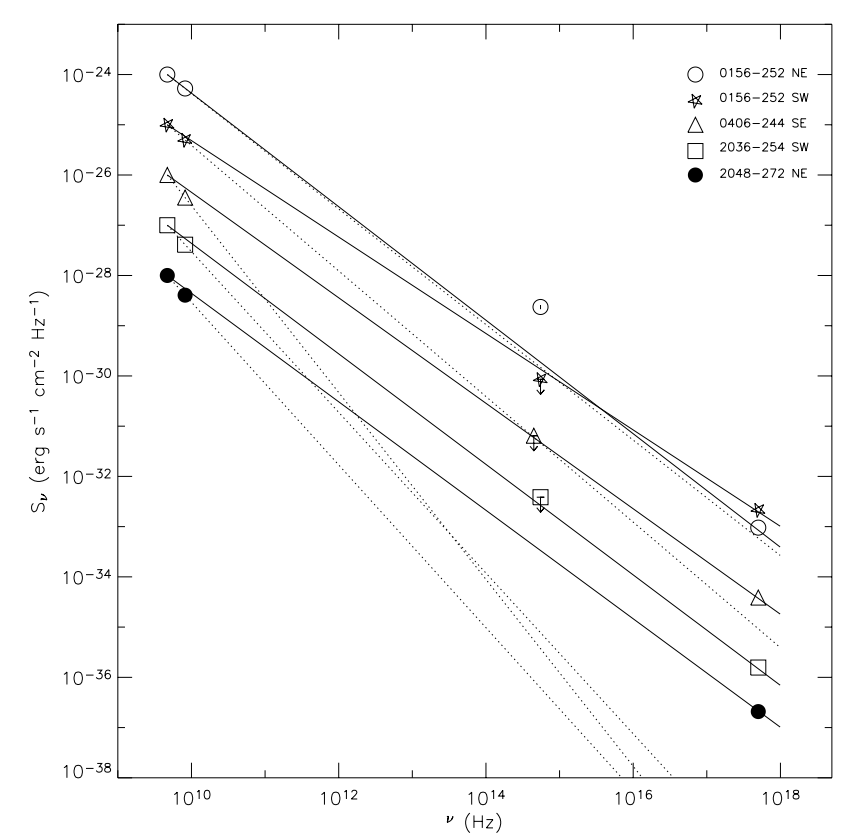

Fig. 4. Synchrotron model predictions. We indicate the observed radio, optical and X-ray flux densities for all components that have a positive $\mathrm{X}$-ray detection. Solid lines represent synchrotron spectra constructed using the $4.7 \mathrm{GHz}$ radio to X-ray spectral index, $\alpha_{4.7}^{\mathrm{X}}$. Dotted lines indicate spectra constructed using a radio spectral index, $\alpha_{4.7}^{8.2}$. For each component, the data and models were offset for clearer visibility of the results. The multiplicative factors for 0156-252 NE, 0156-252 SW, 0406-244 SE, 2036-254 SE and 2048-272 NE were 1.1, 1.4, 0.03, $0.001,0.0003$, respectively.

of frequency. Only a small offset in $\alpha_{4.7}^{8.2}$ can lead to a significant under- or overprediction of the X-ray flux density. The radio spectral indices used were measured over a relatively narrow frequency range, and may be subject to errors. We estimate that the typical error in $\alpha_{4.7}^{8.2}$ is $\sim 0.2$, based on the comparison with a spectral index $\alpha_{1.4}^{8.2}$ determined by combining our data with data (not shown here) from the publicly available NRAO VLA Sky Survey (NVSS) ${ }^{4}$, albeit at much lower resolution. Therefore, we attempt the second method (indicated by solid lines in Fig. 4). We slightly modify the above procedure and now estimate the radio flux density expected at $8.2 \mathrm{GHz}$, assuming a priori that the spectrum is synchrotron with a radio to X-ray spectral index $\alpha_{4.7}^{\mathrm{X}}$. The ratio of predicted to observed $8.2 \mathrm{GHz}$ flux densities is 1.0 for the NE lobe of 0156-252 and ranges from 1.2 to 1.5 for the other lobes. This indicates that the observed X-ray flux densities are consistent with synchrotron radiation, provided that the error in the integrated flux densities at $8.2 \mathrm{GHz}$ are $\gtrsim 20 \%$. Typically, errors in the integrated radio flux densities are of the order of $\sim 5 \%$. Furthermore, the extrapolation from the radio to the $\mathrm{X}$-ray using the radio spectral index under-predicted the observed X-ray emission in all cases. If there were to be a large, random error in the integrated radio flux densities, we might have expected to see some of the spectra over-predict the X-ray flux as well. The fact that this is not observed may indicate that the typical error in the radio flux densities is indeed only a few percent.

\footnotetext{
${ }^{4}$ http://www.cv.nrao.edu/nvss/
}

To further test our synchrotron models, we have extracted flux densities in the optical ( $6000 \AA)$ using the HST/WFPC2 observations of 0156-252, 0406-244, and 2048-272 (see Sect. 2). In Fig. 4 we have indicated $1 \sigma$ upper limits on the optical emission of the SW lobe of 0156-252, the SE lobe of 0406-244 and the NE lobe of 2048-272. Although the flux densities in the optical are consistent with the synchrotron models, they are unfortunately not deep enough to rule them out.

Interestingly, the optical flux density of the NE lobe of 0156-252 is about 1 order of magnitude higher than predicted. We suspect that the optical emission in this lobe is most likely not continuum emission, but line emission. The NE lobe coincides with the peak of the Ly $\alpha$ intensity, and several strong emission lines common to HzRG spectra fall within the filter. Moreover, the HST/WFPC2 image (Fig. 5) shows a bright component roughly following the morphology of the radio lobe, reminiscent of a shell of shocked gas. We will treat this feature in a separate discussion below.

- Synchrotron self-Compton (SSC) emission

SSC emission arises due to Inverse-Compton scattering of local synchrotron photons. This process dominates over other IC mechanisms when the local synchrotron photon energy density is higher than that of the external (e.g. CMB) photon field, and has been found to explain the Chandra hot spots of radio sources such as 3C 295 (Harris et al. 2000) and Cygnus A (Wilson et al. 2000). We calculate the energy density of the local synchrotron photon field, $u_{\mathrm{s}}$, by integrating the radio flux density over 1 decade of frequency $(1-10 \mathrm{GHz})$ and assuming a cylindrical geometry for the radio lobes. The results are listed in Table 5.

A detailed calculation of SSC (see e.g. Band \& Grindlay (1985) for the theoretical framework) would require knowledge of the full radio spectrum and the geometry of lobes and hot spots. However, we can roughly estimate the SSC flux density in the X-ray from the ratio of the energy losses in the IC and synchrotron channels $R=u_{\mathrm{S}} / u_{B} \approx L_{\mathrm{IC}} / L_{\mathrm{S}}$, where $u_{B}$ is the energy density of the magnetic field $B$, and $L_{\mathrm{IC}}$ and $L_{\mathrm{S}}$ are the IC and synchrotron luminosities (see Harris et al. 2000; Donahue et al. 2003). We calculate $u_{B}$ from the magnetic field strength $B_{\text {eq }}$ that will make the energy densities of fields and particles approximately equal. We use the formula given by Miley (1980):

$$
\begin{aligned}
B_{\mathrm{eq}}= & 5.69 \times 10^{-5} \\
& \times\left[\frac{(1+k)}{\eta} \frac{(1+z)^{3+\alpha_{r}} S_{r} v_{r}^{\alpha_{r}}}{\theta^{2} s \sin ^{\frac{3}{2}} \phi} \frac{v_{2}^{\frac{1}{2}-\alpha_{r}}-v_{1}^{\frac{1}{2}-\alpha_{r}}}{\frac{1}{2}-\alpha_{r}}\right]^{\frac{2}{7}},
\end{aligned}
$$

where $B_{\text {eq }}$ is in Gauss, $k$ is the ratio of energy in heavy particles to that in electrons, $\eta$ the filling factor, $\theta^{2}\left(\operatorname{arcsec}^{2}\right)$ the area taken up by the radio lobe (assumed to be a cylinder viewed broadside), $s$ the pathlength through the lobe (kpc), and $v_{1}$ and $v_{2}(\mathrm{GHz})$ are the lower and upper cut-off frequencies of the synchrotron spectrum. We take $\phi=\pi / 2, k=0$ and filling factor unity so as to obtain a real minimum, and note that the results are only weakly dependent on these parameters given the $2 / 7$ power. $R, B_{\text {eq }}$ and $S_{\mathrm{X}, \mathrm{SSC}}$ are given in Table 5 , where 
Table 5. Predictions for SSC and IC/CMB emission, and the equipartition and IC/CMB magnetic field strengths.

\begin{tabular}{|c|c|c|c|c|c|c|c|c|}
\hline Component & Area $^{a}$ & $u_{\mathrm{s}}^{b}$ & $u_{\mathrm{s}} / u_{\mathrm{CMB}}^{c}$ & $R^{d}$ & $f_{0.5-6 \mathrm{keV}, \mathrm{SSC}}^{e}$ & ${\frac{f_{0.5-6 \mathrm{keV}, \text { SSC }}}{f_{0.5-6 \mathrm{keV}, \mathrm{obs}}^{f}}}^{f}$ & $B_{\mathrm{eq}}^{g}$ & $B_{\mathrm{IC} / \mathrm{CMB}}^{h}$ \\
\hline 0156-252 NE & $r=0.35, l=1^{\prime \prime} .0$ & 2.8 & 0.7 & 0.04 & 4.4 & 0.4 & 129 & 104 \\
\hline SW & $r=0 . .^{\prime} 4, l=0 . .7$ & 0.3 & 0.1 & 0.02 & 0.2 & 0.01 & 67 & 30 \\
\hline 0406-244 SE & $r=0^{\prime} \cdot 3, l=1^{\prime \prime} .2$ & 5.6 & 0.9 & 0.05 & 2.7 & 0.2 & 169 & 179 \\
\hline NW & $r=0.3, l=1^{\prime \prime} .2$ & 6.0 & 1.0 & 0.05 & 5.1 & $>0.2$ & 170 & $>130$ \\
\hline $0828+193 \mathrm{NE}$ & $r=0.2, l=0 . \prime 7$ & 7.9 & 1.2 & 0.04 & 0.7 & $>0.04$ & 213 & $>152$ \\
\hline SW & $r=0.2, l=0 . \prime 7$ & 3.3 & 0.5 & 0.03 & 0.4 & $>0.03$ & 162 & $>93$ \\
\hline $2036-254 \mathrm{NE}$ & $r=0,3$ & 2.9 & 0.9 & 0.04 & 2.1 & $>0.1$ & 139 & $>44$ \\
\hline SW & $r=0.3, l=11^{\prime \prime} 0$ & 2.7 & 0.8 & 0.04 & 2.0 & 0.2 & 135 & 135 \\
\hline $2048-272 \mathrm{NE}$ & $r=0.5, l=1^{\prime \prime} .1$ & 3.4 & 0.9 & 0.04 & 6.7 & 0.4 & 126 & 154 \\
\hline SW & $r=0^{\prime} 6, l=2^{\prime \prime} .4$ & 0.3 & 0.1 & 0.02 & 0.3 & $>0.02$ & 61 & $>117$ \\
\hline
\end{tabular}

${ }^{a}$ Cylinder of length $l$ and radius $r$ ( $r$ along the line of sight), or a sphere of radius $r$.

${ }^{b}$ Synchrotron energy density, $u_{\mathrm{S}}$, in units of $10^{-11} \mathrm{erg} \mathrm{cm}^{-3}$.

${ }^{c}$ Ratio of the synchrotron to cosmic microwave background energy densities.

${ }^{d}$ Ratio of the synchrotron to equipartition magnetic field energy densities, $R=u_{\mathrm{S}} / u_{B}$.

${ }^{e}$ Predicted synchrotron self-Compton (SSC) flux in the $0.5-6 \mathrm{keV}$ band in units of $10^{-16} \mathrm{erg} \mathrm{s}^{-1} \mathrm{~cm}^{-2}$.

${ }^{f}$ Ratio of predicted SSC flux to observed X-ray flux.

${ }^{g}$ Equipartition magnetic field strength in $\mu \mathrm{G}$.

${ }^{h} \mathrm{IC} / \mathrm{CMB}$ magnetic field strength in $\mu \mathrm{G}$.

$B_{\text {eq }} \sim 100-200 \mu \mathrm{G}$. In all cases, the ratio of predicted X-ray SSC flux to observed X-ray flux is less than 0.4.

- Inverse-Compton (IC) scattering of the CMB

Another possibility is the up-scattering of CMB photons by relativistic electrons in the radio source. This effect is expected to become significantly more dominant at higher redshifts due to the $(1+z)^{4}$ increase in the energy density of the CMB $\left(u_{\mathrm{CMB}} \sim 3.4 \times 10^{-11} \mathrm{erg} \mathrm{cm}^{-3}\right.$ at $\left.z \sim 2\right)$.

The scattering electrons are assumed to belong to the same power law distribution of electrons which is responsible for the radio synchrotron emission. Therefore, the X-ray flux may be used to constrain the magnetic field strength: since the energy density of the CMB is fixed at any given $z$, less observed X-ray flux implies fewer electrons, so the magnetic field producing the observed radio synchrotron emission must be stronger. Using the formalism of Harris \& Grindlay (1979) we calculate the magnetic field strength, $B_{\mathrm{IC}}$ :

$B_{\mathrm{IC}}^{1+\alpha_{r}}=\left[\frac{\left(5.05 \times 10^{4}\right)^{\alpha_{r}} C\left(\alpha_{r}\right) G\left(\alpha_{r}\right)(1+z)^{3+\alpha_{r}} S_{r} v_{r}^{\alpha_{r}}}{10^{47} S_{\mathrm{X}} v_{\mathrm{X}}^{\alpha_{r}}}\right]$,

where $B_{\mathrm{IC}}$ is in Gauss, $\alpha_{r}$ is the radio spectral index, $S_{r}$ and $S_{\mathrm{X}}$ are the radio and X-ray flux densities $\left(\mathrm{erg} \mathrm{s}^{-1} \mathrm{~cm}^{-2} \mathrm{~Hz}^{-1}\right)$ at $v_{r}$ and $v_{\mathrm{X}}$ (both in $\mathrm{Hz}$ ), respectively. $C\left(\alpha_{r}\right)$, which is well approximated by the value $1.15 \times 10^{31}$, and $G\left(\alpha_{r}\right)$, which is a function that is slowly varying with $\alpha_{r}$, can be found in Harris \& Grindlay (1979). From the radio and X-ray flux densities listed in Table 4 we derive field strengths of $\sim 30-180 \mu \mathrm{G}$ for the components that are detected in the X-ray. The results are listed in Table 5, where we also give lower limits on $B_{\mathrm{IC}}$ for the undetected lobes.

We can compare $B_{\text {IC }}$ to $B_{\text {eq }}$, which is an estimate of the magnetic field that is solely based on the observed radio synchrotron flux (see expression above). The $B_{\text {eq }}$ field strengths are typically $\sim 100-200 \mu \mathrm{G}$, remarkably close to the field strengths derived for the IC/CMB mechanism. The general agreement that we find between the magnetic field strengths using the two independent field estimators is consistent with the X-ray flux being produced by the IC/CMB process.

The (observed) 1-10 GHZ synchrotron flux in HzRGs is produced by relativistic electrons that have $\gamma \sim 10^{3.4-3.9}$ for $B \sim 100 \mu \mathrm{G}\left(v_{\mathrm{syn}}=4.2(B / 1 \mu \mathrm{G}) \gamma^{2} \mathrm{~Hz}\right.$, see Bagchi et al. 1998). The number density of relativistic electrons with energies between $\gamma$ and $\gamma+\mathrm{d} \gamma$ can be expressed as a power-law, $N(\gamma) \mathrm{d} \gamma=N \gamma^{-s} \mathrm{~d} \gamma$ with $s=2 \alpha+1$. The up-scattering of $\mathrm{CMB}$ photons to $\mathrm{X}$-ray frequencies is provided by electrons with a Lorentz factor $\gamma \sim 10^{3}$, since $v_{\text {out }} \approx \gamma^{2} v_{\text {in }}$, and the frequency for which the energy density of the CMB peaks is $\sim 1.6 \times 10^{11} \times(1+z) \mathrm{Hz}$. Electrons having such Lorentz factors are highly abundant, given the extrapolation of $N(\gamma)$ to $\gamma \sim 10^{3}$. Note that in the SSC process described above, the $\mathrm{X}$-ray emission must be produced by the up-scattering of synchrotron photons off a population of relativistic electrons having $\gamma \sim 10^{4-4.5}$. Since $N(\gamma)$ decreases strongly with increasing $\gamma$ and $\alpha>1$ for all our sources, $N\left(10^{4.5}\right)$ will be several orders of magnitude lower than $N\left(10^{3}\right)$. Together with the fact that $u_{\mathrm{s}} / u_{\mathrm{CMB}}$, is around unity (see Table 5 ) this implies that the IC/CMB process will be a far more efficient process for producing X-rays at these redshifts. While the contribution of SSC to the observed X-ray flux might still be in the range of $\sim 1-40 \%$ as detailed above, the SSC output is expected to peak around $10^{15} \mathrm{~Hz}$, or in the UV part of the spectrum.

- Other mechanisms

The ambient medium in which a radio source expands is expected to be very different for HzRGs compared to radio sources at low redshift. Low redshift sources often lie in a smooth, virialised $\left(T \sim 10^{8} \mathrm{~K}\right)$ atmosphere. For HzRGs such a smooth medium does probably not exist because the necessary potential well has not yet formed. Instead they lie in a 
multiphase medium, consisting of cold $\left(10^{4} \mathrm{~K}\right)$, high density clouds embedded in low density regions approaching virial temperatures $\left(10^{6-7}\right)$. The passage of a radio jet through the multiphase can lead to interesting phenomena, such as jetinduced star formation in the high density clouds and shock heating of lower density regions. When intermediate density regions are shocked they may cool off, emitting strong emission lines such as $\operatorname{Ly} \alpha$. The lower density gas with high filling factor will be shock heated to temperatures where the gas starts producing X-rays. Can the X-ray emission coincident with the NE lobe of $0156-252$ be produced by shocks in this thermal gas? Interestingly, the NE radio lobe of 0156-252 coincides with the peak of the Ly $\alpha$ emission, and the HST/WFPC2 image (see Fig. 5) shows evidence for a shell of shocked (emissionline) gas that follows the bend of the radio lobe.

For 1138-262, Carilli et al. (2002) propose that much of the extended X-ray emission comes from ambient gas that is shock heated by the expanding radio source. For this gas they estimate a density of $0.05 \mathrm{~cm}^{-3}$ and a pressure of $10^{-9} \mathrm{dyn} \mathrm{cm}^{-2}$. This pressure is comparable to the optical line emitting gas and to minimum pressures in the radio source, with a total gas mass of about $2.5 \times 10^{12} M_{\odot}$. Carilli et al. hypothesize that the high filling factor X-ray emitting gas may confine both the radio source and the line emitting clouds. The extended X-ray luminosity associated with the NE lobe of $0156-252$ is a factor 5 or so less than in 1138-262, implying a factor 5 lower total gas mass (for a fixed density) if the X-rays represent shocked gas.

So far, the discussion has been limited to the X-ray emission directly coincident with the radio lobes and hotspots. Brunetti (2000) describes a mechanism in which IC scattering of (mostly infrared) AGN photons by relativistic electrons can also produce X-ray bright emission. Such a mechanism, if it exists, may contribute significantly to the radio/X-ray alignment effect. Under the Brunetti mechanism the X-ray luminosity is expected to decrease with increasing distance from the source (i.e. the hidden quasar) producing the photons. This will result in opposite gradients in the X-ray and radio luminosities, and entails (in our case) the presence of an inner, undetected part of the radio lobe. Brunetti (2000) predicts that the receding radio lobe will produce brighter X-ray emission than the approaching lobe, because time delay makes it closer to the nucleus and because backward scattering is more effective than forward scattering. In 0406-244 (and possibly in one or several of the other sources as well) there is X-ray emission in between the core and the SE lobe. The nature of this emission could be similar to the aligned emission seen in several other high-redshift quasars and radio galaxies (e.g. Yuan et al. 2003; Fabian et al. 2003; Scharf et al. 2003) for which the Brunetti model is among the possible scenarios. However, the proposed mechanism relies on a (suggested) supply of electrons with $\gamma \lesssim 100$. Given the highly speculative amplitudes at these low energies, we will not pursue this mechanism quantitatively.

\subsection{Thermal emission from hot (cluster) gas}

An important driver for observing powerful HzRGs in the $\mathrm{X}$-ray is to search for traces of thermal emission from an ICM.
To test whether our data show any evidence for the presence of extended, diffuse X-ray emission we will attempt two methods:

\section{- Smoothed fields}

To obtain upper limits on the luminosity of extended regions in each field, we place 10 circles of 12" diameter (corresponding to $\sim 100 \mathrm{kpc}$ at $z \sim 2$ ) around each radio source in regions without visible point sources. We calculate the average background count in these regions and the $1 \sigma$ deviation from the mean. We use the resulting $5 \sigma$ countrates to calculate $5 \sigma$ upper limits on the X-ray flux of typical $100 \mathrm{kpc}$-sized areas. We assumed a Raymond-Smith thermal spectrum with $k T=1 \mathrm{keV}$, and used the galactic $n_{\mathrm{HI}}$ for each source to produce the unabsorbed flux at $0.2-6 \mathrm{keV}$. We derive an upper limit on the flux of (4-9) $\times 10^{-15} \mathrm{erg} \mathrm{cm}^{-2} \mathrm{~s}^{-1}$, corresponding to luminosities of $(1.5-4) \times 10^{44} \mathrm{erg} \mathrm{s}^{-1}$ (for a uniform sphere).

A $5 \sigma$ deviation from the background countrate over a $12^{\prime \prime}$ region would easily stand out from the X-ray maps after smoothing with a large kernel. Therefore, we create smoothed images of diffuse emission. First, we remove counts that are associated with the radio galaxy by replacing all counts in a circular region encompassing the entire radio structure by the background using the task DMFILTH that maintains the Poissonian nature of the background. The background was estimated from a large annular region around the radio source. We then run the WAVDETECT algorithm (Freeman et al. 2002) within CIAO to identify the remaining point sources and replace them by the background. The images were smoothed by a $10^{\prime \prime}(F W H M)$ Gaussian. The images were then visually inspected to look for regions of diffuse emission. We found a single detection of an extended X-ray component in the smoothed map of 0156-252 shown in Fig. 6. In an ovally shaped region that can be approximated by an ellipse of $10^{\prime \prime} \times 17^{\prime \prime}$ $(83 \times 142 \mathrm{kpc}$ if it were to be located at the same distance as the radio galaxy), we find $14.4 \pm 5$ net counts $(3 \sigma)$ in the energy range (0.2-6) keV with an average energy of $1.2 \mathrm{keV}$. The centroid of the region is situated approximately $12^{\prime \prime}$ to the northeast of the core of the radio source. The (observed-frame) $0.5-2 \mathrm{keV}$ unabsorbed flux is $2.3 \times 10^{-15} \mathrm{erg} \mathrm{cm}^{-2} \mathrm{~s}^{-1}$. If this emission were to come from a sphere of thermal gas at the redshift of the radio source, it would correspond to a luminosity of $7.4 \times 10^{43} \mathrm{erg} \mathrm{s}^{-1}$ and a mass of $10^{11} M_{\odot}$ (assuming a uniform sphere). We could not identify this X-ray region with objects in any of our optical and near-infrared imaging data. Therefore, in this particular case the true nature of the emission remains highly obscure, and we cannot rule out it being due to noise. However, it illustrates the kind of emission that might be detectable in the search for high redshift cluster gas, provided a proper identification can be made.

\section{- Stacked fields}

To further search for emission from thermal gas in an annular region surrounding the radio sources, we stacked the five $20 \mathrm{ks}$ exposures to obtain a single $100 \mathrm{ks}$ exposure of a typical HzRG field. Each field was cleared of point sources before stacking them. In the stacked image, we then replaced the entire region inside a circle encompassing the largest of the radio sources by the background to ensure that we are not measuring extended X-ray emission associated with the radio structures. 


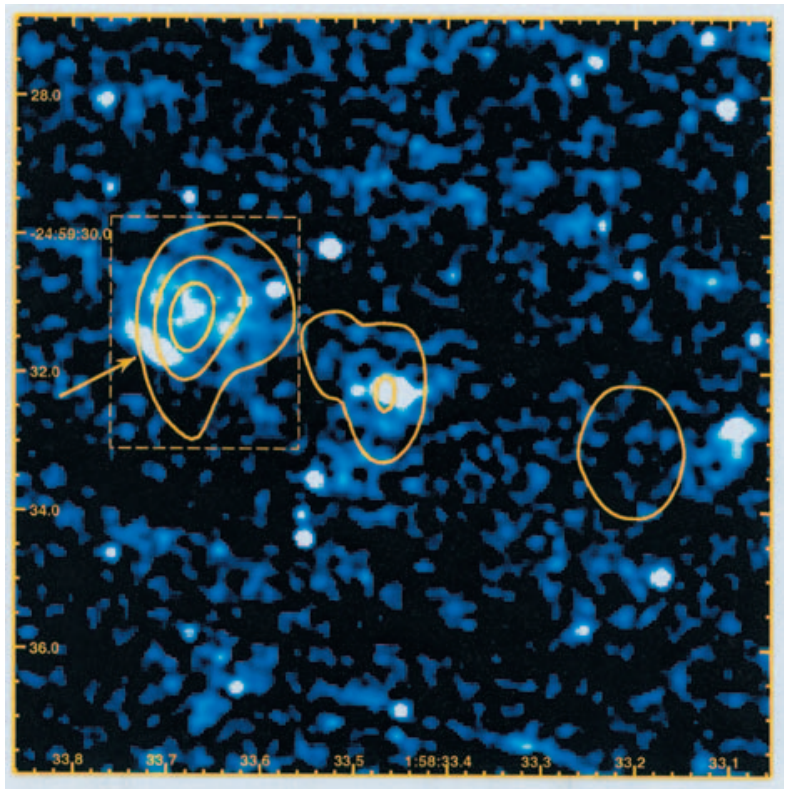

Fig. 5. HST/WFPC2 F555W image of 0156-252 with $4.7 \mathrm{GHz}$ VLA radio contours superimposed. We have detected extended UV line emission (most likely CIII] $\lambda 1909 \AA$ A) at the position of the NE lobe (box region). An elongated feature in the extended emission resembles a bow-shock that follows the radio lobe (arrow).

The stacked image was smoothed by a $10^{\prime \prime}$ (FWHM) Gaussian. The stacked image and the smoothed image are shown in Fig. 7. The region used for the background determination is indicated by the large, dashed annulus. The small circle in the center of the field indicates the size of the largest radio source. From the smoothed image, where the scales run from $2 \sigma$ below the mean to $2 \sigma$ above the mean, we see no evidence for diffuse, extended emission in the vicinity of the radio source(s). We obtained a radial profile of surface brightness from the stacked image using the annuli as indicated in Fig. 7. The radial profile out to $1^{\prime}$ is consistent with zero contribution from diffuse, extended X-ray emission, as shown in Fig. 8. Out to a radius of $\sim 75^{\prime \prime}(\sim 625 \mathrm{kpc})$ we measure $25 \pm 85$ counts. Assuming a $1 \mathrm{keV}$ thermal spectrum and correcting for galactic absorption, the $3 \sigma$ upper limit on the (observed) $0.2-6 \mathrm{keV}$ flux inside this radius is $1.2 \times 10^{-14} \mathrm{erg} \mathrm{cm}^{-2} \mathrm{~s}^{-1}$, corresponding to a luminosity of $<4 \times 10^{44} \mathrm{erg} \mathrm{s}^{-1}$. We derive a central electron density of $<7 \times 10^{-3} \mathrm{~cm}^{-3}$ and a total enclosed gas mass of $<4 \times 10^{13} M_{\odot}$, assuming an isothermal sphere with a cluster $\beta$-model surface brightness profile with $\beta=0.67$ and a core radius of $200 \mathrm{kpc}$.

\subsection{Serendipitous $X$-ray sources}

Pentericci et al. (2002) found evidence of an excess of Chandra detected X-ray sources in the vicinity of radio galaxy PKS $1138-262$ at $z=2.16$. They found 16 sources in the soft band $(0.5-2 \mathrm{keV})$ with a minimum flux of $1 \times$ $10^{-15} \mathrm{erg} \mathrm{cm}^{-2} \mathrm{~s}^{-1}$, compared to 10.3 (11) calculated from the Chandra Deep Field South (North) for a similarly sized region, and 8 sources with fluxes $\geq 3 \times 10^{-15} \mathrm{erg} \mathrm{cm}^{-2} \mathrm{~s}^{-1}$ compared to 4 (5.1) for the Chandra Deep Fields. Although this may not be considered a substantial overdensity in angular space

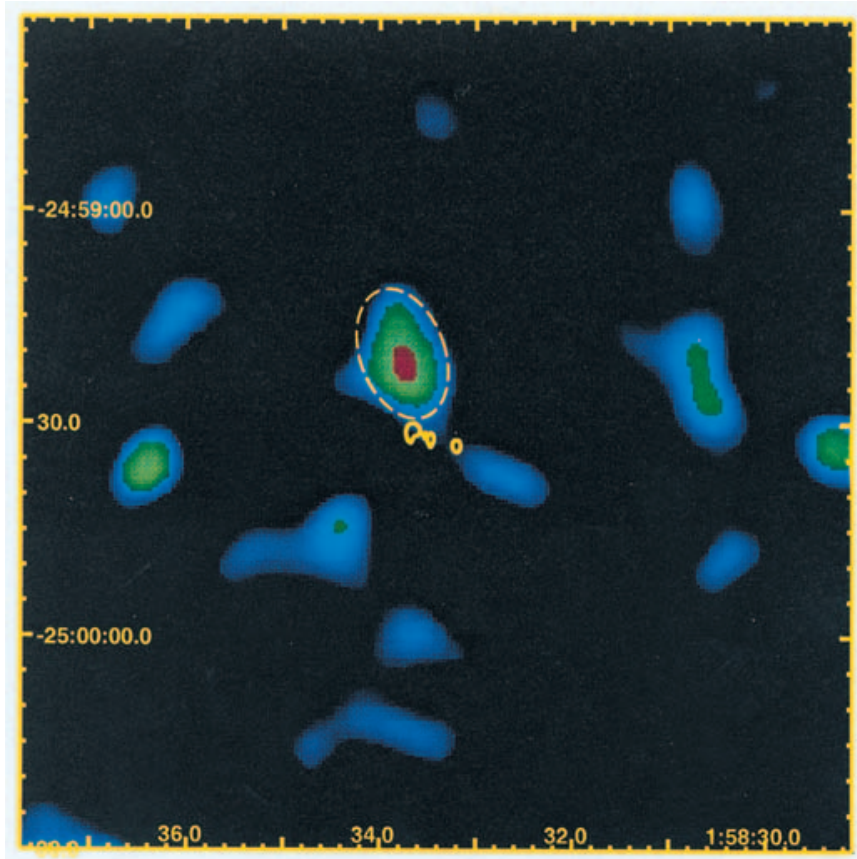

Fig. 6. X-ray image of the field around 0156-252 . The image was smoothed by a Gaussian $\left(10^{\prime \prime} F W H M\right)$, after replacing the X-ray counts due to the radio source and point sources in the field by a Poissonian background. A large region of diffuse emission (indicated by the dashed ellipse) is found just northeast to the radio galaxy (indicated by its radio contours).

(an excess of $\sim 50 \%$ compared to a typical cosmic variance of $20-30 \%$ ), it is very significant in redshift space given that six of the serendipitous X-ray sources could be identified with previously discovered $\mathrm{Ly} \alpha$ or $\mathrm{H} \alpha$ emitting galaxies in the protocluster surrounding PKS 1138-262 (Pentericci et al. 2002). Therefore, observing overdensities of X-ray sources may provide evidence for the existence of galaxy overdensities associated with radio sources (see also Cappi et al. 2001).

Following Pentericci et al. (2002), we use WAVDETECT to investigate the presence of serendipitous sources in our 5 fields. Because the 2 separate observations of 0156-252 were taken at different roll angles, the corners of the ACIS-S3 chip only have half of the total $20 \mathrm{ks}$ exposure time. We specify a circular region covering the maximum area having the full $20 \mathrm{ks}$ depth. The circle has a radius of $3.852^{\prime}$, thereby covering an area of $46.62^{\prime 2}$. For consistency we use this region in all the fields. We limit the energy range for source detection to a maximum of $2 \mathrm{keV}$ (cf. Pentericci et al. 2002) to improve the $S / N$ (the background is lower at lower energies). For each field, we exclude the radio source, and we only consider sources that are detected with $S / N>3$, where we define $S / N$ as the number of background-subtracted counts divided by the root of the total counts. The net count rates of the detected sources were converted into fluxes assuming a power-law spectrum with Galactic absorption along the line of sight, and a photon index of $\Gamma=2.0$ for the soft band. Table 6 lists the number of sources in each field that have soft $(0.5-2 \mathrm{keV})$ fluxes of $>1.5$ and $>3 \times 10^{-15} \mathrm{erg} \mathrm{cm}^{-2} \mathrm{~s}^{-1}$. Besides the number of sources found in each $46.62^{\prime 2}$ area we give the number density per 

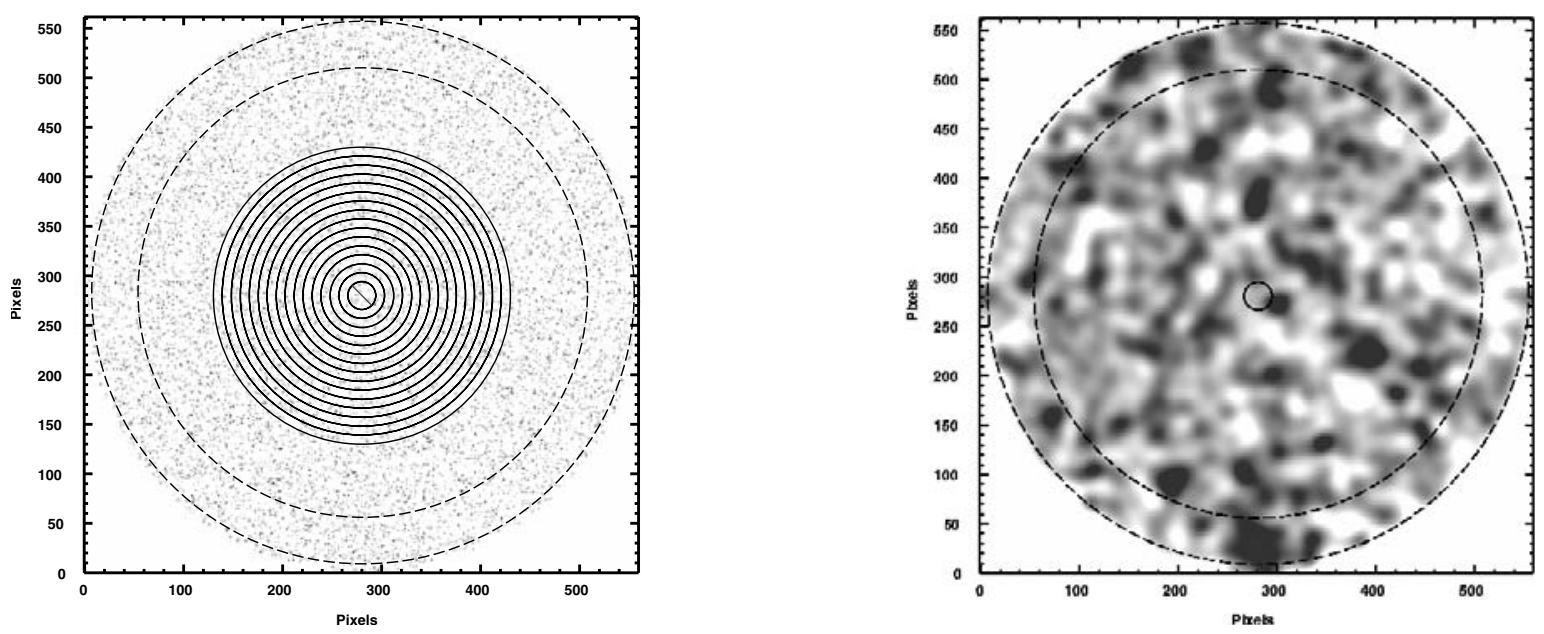

Fig. 7. Left: stacked image of the five HzRG fields, after subtraction of point sources. Indicated are the annuli from which a radial surface brightness profile was extracted. The innermost circle encompasses the total extent of the largest radio source in the sample. Counts inside this region due to the radio sources have been replaced by the background, estimated from the large, dashed annular region. Right: stacked image smoothed by a $10^{\prime \prime}$ ( $F W H M$ ) Gaussian. Scales run from $2 \sigma$ below (white) to $2 \sigma$ above (black) the mean. The background region and the maximum size of the radio sources are indicated. The distinct peaks in this summed image are not traced back to significant features in any of the individual images.

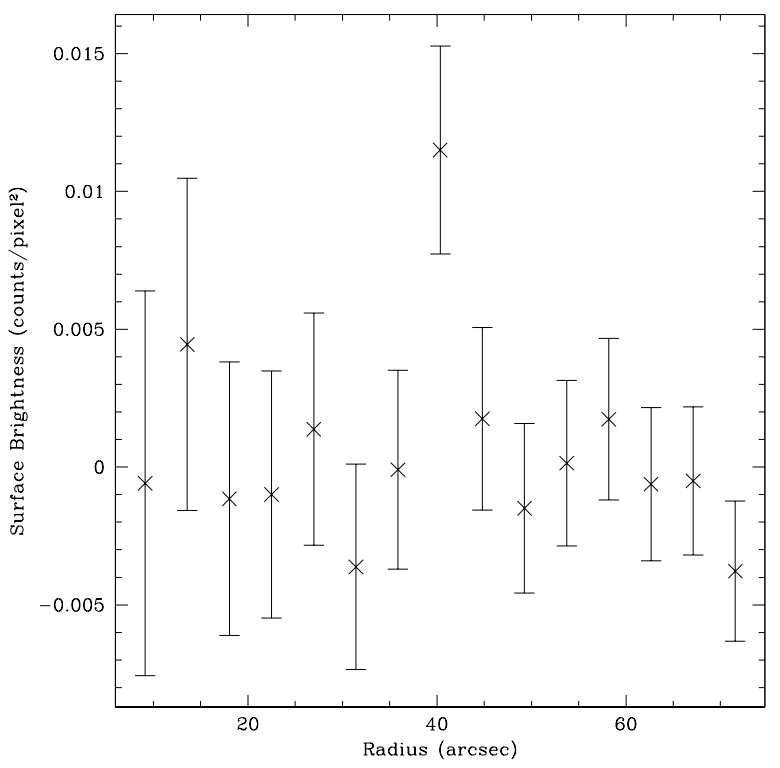

Fig. 8. Radial surface brightness profile extracted from the stacked image shown in Fig. 7.

square degree between parentheses. We also list the number of sources in each bin averaged over the 5 fields. For comparison, we have run the same detection procedure on the observations of PKS 1138-262 using (i) the full $40 \mathrm{ks}$ exposure; and (ii) a $20 \mathrm{ks}$ subsample. We have also indicated the number of sources found in the Chandra Deep Fields (Mushotzky et al. 2000; Giacconi et al. 2001).

The number of bright sources in the field of 0406-244 is twice as high compared to the Chandra Deep Fields, and similarly high as PKS 1138-262. The number of sources in the field of 2048-272 is less than half of the number of sources in the CDFs in both flux bins. On average we find 6 and 4 sources in the $>1.5$ and $>3 \times 10^{-15} \mathrm{erg} \mathrm{cm}^{-2} \mathrm{~s}^{-1}$ flux bins,
Table 6. The number of X-ray sources detected in the fields of $z \sim 2$ radio galaxies, and comparison with the field population expected based on the Chandra deep fields.

\begin{tabular}{lcc}
\hline \hline Field & \multicolumn{2}{c}{ Number of sources $^{a}$} \\
& $f_{0.5-2 \mathrm{keV}}^{b}>1.5$ & $f_{0.5-2 \mathrm{keV}}^{b}>3.0$ \\
\hline $0156-252$ & $7(541)$ & $5(386)$ \\
$0406-244$ & $7(541)$ & $7(541)$ \\
$0828+193$ & $5(386)$ & $4(309)$ \\
$2036-254$ & $7(541)$ & $5(386)$ \\
$2048-272$ & $2(154)$ & $1(77)$ \\
average of fields & $6(463)$ & $4(309)$ \\
\hline $1138-262^{c}$ & $7(541)$ & $6(463)$ \\
$1138-262^{d}$ & $11(715)$ & $7(541)$ \\
CDFs $^{e}$ & $6 / 7(470 / 540)$ & $3 / 4(260 / 330)$ \\
\hline
\end{tabular}

${ }^{a}$ Number of X-ray sources sources found in the field (per $\mathrm{deg}^{2}$ ).

${ }^{b}$ Minimum source flux in units of $10^{-15} \mathrm{erg} \mathrm{s}^{-1} \mathrm{~cm}^{-2}$.

${ }^{c}$ Comparison 1: A $20 \mathrm{ks}$ subsample of the full $40 \mathrm{ks}$ observation of $1138-262$.

${ }^{d}$ Comparison 2: The full 40 ks exposure of 1138-262 (Pentericci et al. 2002).

${ }^{e}$ Comparison 3: The Chandra Deep Field South/North (Mushotzky et al. 2000; Giacconi et al. 2001).

respectively, in good agreement with the CDFs. Based on these number counts of serendipitous sources, we find no evidence that the radio sources lie in the same cluster environments as is observed in the case of PKS 1138-262 by Pentericci et al. (2002). However, as indicated in Table 6 the factor $\sim 2$ excess of faint, $>3 \sigma$ sources in the field of PKS 1138-262 only becomes apparent in the full $40 \mathrm{ks}$ exposure, which may indicate that our exposures are not deep enough to make a good comparison.

To further study whether there is a preference for field sources to lie in the vicinity of high-redshift radio sources, 


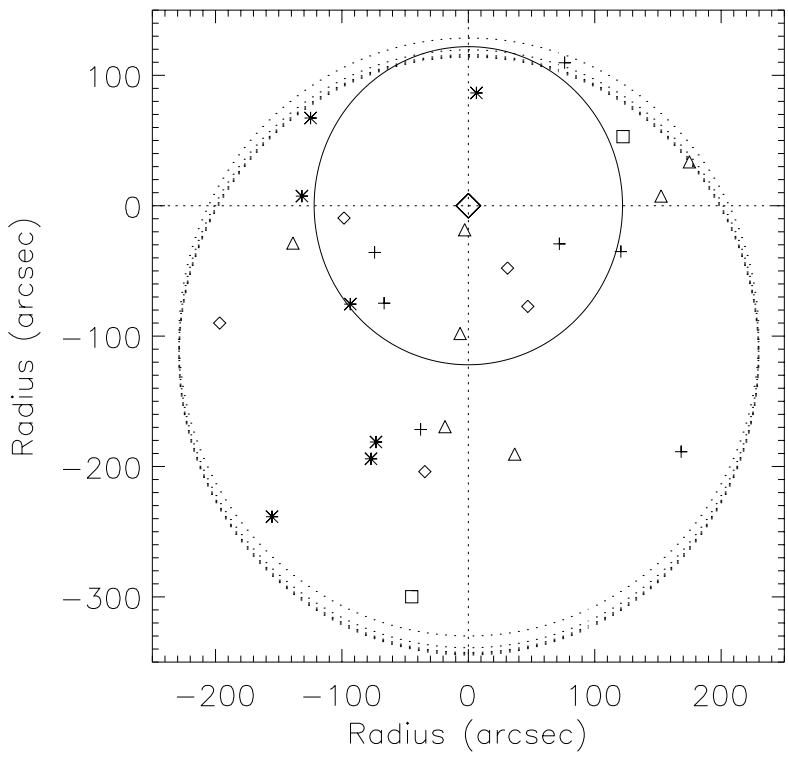

Fig. 9. The positions of serendipitous $X$-ray sources with flux $>1.5 \times$ $10^{-15} \mathrm{erg} \mathrm{cm}^{-2} \mathrm{~s}^{-1}$ in the five HzRG fields combined. Different symbols indicate the sources in the 5 individual fields. The five images are aligned so that the radio sources (indicated by large diamond) all coincide with the position of 0156-252, and rotated to maximize the overlapping areas of the different pointings (indicated by dotted circles). The full circle indicates an area of $1 \mathrm{Mpc}$ in radius around the radio source.

we register the five fields using the radio source positions as centroids, and we rotate the fields around their original center so that the area of overlap is maximized. This is shown in Fig. 9. The number density of sources found within $1 \mathrm{Mpc}$ (at $z \sim 2$ ) from the radio sources is $0.77 \mathrm{arcmin}^{-2}$, compared to $0.4 \mathrm{arcmin}^{-2}$ for the density of sources outside this region and $0.6 \operatorname{arcmin}^{-2}$ for the average density of the entire field. However, the cosmic variance of field X-ray sources is significant (25\%, e.g. Cappi et al. 2001), making it impossible to conclude if serendipitous sources cluster around radio sources on the basis of the current data. Spectroscopy may confirm whether some of these sources are associated with the radio galaxies.

\section{Summary and conclusions}

We have studied X-ray observations of five radio galaxies at $2<z<2.6$, thereby significantly increasing the number of Chandra studies on high redshift radio galaxies. The main conclusions from our analysis are the following.

The X-ray emission that we detect from the nuclei are consistent with obscured power-law spectra as observed for powerful radio galaxies over a wide redshift range (e.g. Harris et al. 2000; Carilli et al. 2002; Young et al. 2002; Hardcastle et al. 2002; Scharf et al. 2003), and can be explained by the unified model in which the broad-line region of radio galaxies is obscured by a dusty torus surrounding the nucleus. For 0156-252, this conclusion is confirmed by the fact that the best-fit core spectrum is an absorbed power-law with $n(\mathrm{HI}) \sim$ $2 \times 10^{22} \mathrm{~cm}^{-2}$.
Extended X-ray emission coincident with the radio lobes was detected for several of the sources, albeit at low $S / N$. For all but one source, the straight extrapolation from the radio to the X-ray using the radio spectral index rules out a synchrotron origin of the emission, unless there are large errors in the radio spectral indices measured between 5 and $8 \mathrm{Ghz}$. Although the predicted X-ray synchrotron flux in source 0156-252 is close to the observed value, we have found evidence for the $\mathrm{X}$-ray emission being likely associated with shocked, line emitting gas. Our observations confirm that the radio lobes of these high redshift sources may interact with the surrounding (forming) IGM.

We interpret the X-ray emission of the remaining sources as being due to the inverse Compton scattering of CMB photons off radio synchrotron electrons. This conclusion is supported by the fact that our estimates of the IC/CMB and equipartition magnetic field strenghts are in good agreement. Although IC/CMB is usually dominated by other processes (e.g. SSC) at low redshift, the relative ease with which it is detected in these HzRGs may be ascribed to the $(1+z)^{4}$ increase in the energy density of the CMB.

This research was partly motivated by the large $R M$ observed for a significant fraction of HzRGs. Can the large $R M$ be caused by cluster-sized atmospheres surrounding the radio sources? Taking reasonable estimates for the pathlength $(\sim 100 \mathrm{kpc})$ and the cluster magnetic field strength $(\sim 10 \mu \mathrm{G})$, an intrinsic $R M$ of $\sim 1000 \mathrm{rad} \mathrm{m}^{-2}$ would require an ICM electron density of $\sim 1 \times 10^{-3} \mathrm{~cm}^{-3}$. The existence of cores much denser than this is unlikely, since they would have an X-ray luminosity of $\gtrsim 10^{44} \mathrm{erg} \mathrm{s}^{-1}$ which has not been observed in any of the HzRGs observed to this date. Although higher density material $\left(n_{\mathrm{e}} \sim 200 \mathrm{~cm}^{-3}\right)$ is present in many HzRGs in the form of $10^{4} \mathrm{~K}$ emission line clouds, the filling factor of this gas $\left(f_{\mathrm{e}} \sim 10^{-6}\right)$ usually implied makes it hard to reproduce the high values of $R M$ (e.g. Pentericci 1999). Alternatively, the large $R M$ can arise from the radio emission passing through a sheath of shocked gas surrounding the radio lobes (e.g. Athreya et al. 1998; Carilli et al. 2002, and references therein). The increased gas density implied can then explain the large $R M$ if the magnetic field is ordered on scales of only a few kpc. If the X-ray emission from the NE lobe of 0156-252 is from shocked gas (as suggested by the bow shock feature seen in the HST/WFPC2 image, see Fig. 5), then $B \sim 13 \mu \mathrm{G}$ given the $R M$ from Table 2 and the derived density of $0.05 \mathrm{~cm}^{-3}$.

The existence of protoclusters around several HzRGs at $z \sim 2$ and higher has been established, mainly through narrowband, Ly $\alpha$ imaging observations and spectroscopy. However, such studies have not yet been carried out for the sources presented in this paper. An overdensity of X-ray sources associated with the well-known galaxy protocluster around the radio source 1138-262 at $z=2.2$ was found by Pentericci et al. (2002). We have analysed the number density of X-ray sources in each of the five Chandra fields. None of the fields showed evidence of large-scale structure associated with the radio sources. This may suggest that these HzRGs are not found in the same, overdense environments as 1138-262. Similarly, employing several different methods we found no evidence for virialised gas, although the upper limits that we derive on 
thermal gas are not inconsistent with a direct, no-evolution extrapolation of local X-ray luminous clusters out to $z \sim 2$. Based on the X-ray observations presented in this paper alone, the current standing is that these five HzRGs are not in galaxy overdensities. However, we remark that X-ray observations are not the most effective way to search for galaxy overdensities given the relatively small number fraction of AGN expected at each particular epoch.

Recent observations of some of the most distant, X-ray luminous clusters made with the Advanced Camera for Surveys aboard HST, show a color-magnitude relation comparable to that of local clusters, indicating that early-type galaxies were already well-established by $z \sim 1$ (Blakeslee et al. 2003). Thus, the epoch in which the global relations that exist in clusters today are shaped must probably be sought at significantly higher redshifts than currently probed (i.e. $z \gtrsim 1.3$ ). About a dozen high redshift galaxy overdensities or protoclusters have now been found at $z \gtrsim 2$, either around HzRGs and quasars or in wide-field surveys. In the near future we may expect several candidates suitable for a detailed study with Chandra that could determine exactly at what redshift the virialised gas was established. However, the sensitivity required may be of the order of that of the Chandra deep fields, due to the extreme cosmological surface brightness dimming. While HzRGs have so far presented the best evidence of being associated with massive, forming clusters, they usually come with a plethora of nonthermal X-ray mechanisms. Especially if the radio sources lie at the bottom of the potential well that coincides with the peak of the bremsstrahlung luminosity, they may not be ideal targets for trying to detect this extemely low surface brightness emission from the ICM.

Acknowledgements. The work at SAO was partially supported by NASA grant GO2-3139B and contract NAS8-39073. We thank Michiel Reuland and Andrew Zirm for productive discussions, and Melanie Johnston-Hollitt for helping out with the MIRIAD software. We are grateful to the referee, P. Tozzi, for his many good comments.

\section{References}

Athreya, R. M., Kapahi, V. K., McCarthy, P. J., \& van Breugel, W. 1998, A\&A, 329, 809

Bagchi, J., Pislar, V., \& Lima Neto, G. B. 1998, MNRAS, 296, L23

Band, D. L., \& Grindlay, J. E. 1985, ApJ, 298, 128

Belsole, E., Worrall, D. M., Hardcastle, M. J., Birkinshaw, M., \& Lawrence, C. R. 2004, MNRAS, 352, 924

Blakeslee, J. P., Franx, M., Postman, M., et al. 2003, ApJ, 596, L143

Brand, K., Rawlings, S., Hill, G. J., et al. 2003, MNRAS, 344, 283

Brinkmann, W., Laurent-Muehleisen, S. A., Voges, W., et al. 2000, A\&A, 356, 445

Brunetti, G. 2000, Astropart. Phys., 13, 107

Cappi, M., Mazzotta, P., Elvis, M., et al. 2001, ApJ, 548, 624

Carilli, C. L., Harris, D. E., Pentericci, L., et al. 2002, ApJ, 567, 781

Carilli, C. L., Roettgering, H. J. A., van Ojik, R., Miley, G. K., \& van Breugel, W. J. M. 1997, ApJS, 109, 1

Carilli, C. L., \& Taylor, G. B. 2002, ARA\&A, 40, 319

Dickey, J. M., \& Lockman, F. J. 1990, ARA\&A, 28, 215

Donahue, M., Daly, R. A., \& Horner, D. J. 2003, ApJ, 584, 643

Eilek, J. A., \& Owen, F. N. 2002, ApJ, 567, 202
Ensslin, T. A., Vogt, C., Clarke, T. E., \& Taylor, G. B. 2003, ApJ, 597, 870

Fabian, A. C., Crawford, C. S., \& Iwasawa, K. 2002, MNRAS, 331, L57

Fabian, A. C., Sanders, J. S., Crawford, C. S., \& Ettori, S. 2003, MNRAS, 341, 729

Gehrels, N. 1986, ApJ, 303, 336

Giacconi, R., Rosati, P., Tozzi, P., et al. 2001, ApJ, 551, 624

Hardcastle, M. J., Birkinshaw, M., Cameron, R. A., et al. 2002, ApJ, 581,948

Hardcastle, M. J., \& Worrall, D. M. 1999, MNRAS, 309, 969

Harris, D. E., \& Grindlay, J. E. 1979, MNRAS, 188, 25

Harris, D. E., Nulsen, P. E. J., Ponman, T. J., et al. 2000, ApJ, 530, L81

Ivison, R. J., Dunlop, J. S., Smail, I., et al. 2000, ApJ, 542, 27

Iwamuro, F., Motohara, K., Maihara, T., et al. 2003, ApJ, 598, 178

Kurk, J. 2003, Ph.D. Thesis, Universiteit Leiden

Kurk, J. D., Röttgering, H. J. A., Pentericci, L., et al. 2000, A\&A, 358, L1

Leahy, J. P. 1987, MNRAS, 226, 433

McCarthy, P. J., Baum, S. A., \& Spinrad, H. 1996, ApJS, 106, 281

Miley, G. 1980, ARA\&A, 18, 165

Miley, G. K., Overzier, R. A., Tsvetanov, Z. I., et al. 2004, Nature, 427, 47

Mushotzky, R. F., Cowie, L. L., Barger, A. J., \& Arnaud, K. A. 2000, Nature, 404, 459

Pentericci, L. 1999, Ph.D. Thesis

Pentericci, L., Kurk, J. D., Carilli, C. L., et al. 2002, A\&A, 396, 109

Pentericci, L., Kurk, J. D., Röttgering, H. J. A., et al. 2000a, A\&A, 361, L25

Pentericci, L., McCarthy, P. J., Röttgering, H. J. A., et al. 2001, ApJS, 135, 63

Pentericci, L., Röttgering, H. J. A., Miley, G. K., et al. 1999, A\&A, 341, 329

Pentericci, L., Van Reeven, W., Carilli, C. L., Röttgering, H. J. A., \& Miley, G. K. 2000b, A\&AS, 145, 121

Perley, R. A., \& Taylor, G. B. 1991, AJ, 101, 1623

Rosati, P., Stanford, S. A., Eisenhardt, P. R., et al. 1999, AJ, 118, 76

Rosati, P., Tozzi, P., Ettori, S., et al. 2004, AJ, 127, 230

Rudnick, L., \& Blundell, K. M. 2003, ApJ, 588, 143

Rush, B., McCarthy, P. J., Athreya, R. M., \& Persson, S. E. 1997, ApJ, 484,163

Scharf, C., Smail, I., Ivison, R., et al. 2003, ApJ, 596, 105

Simard-Normandin, M., \& Kronberg, P. P. 1980, ApJ, 242, 74

Smail, I., Scharf, C. A., Ivison, R. J., et al. 2003, ApJ, 599, 86

Smith, D. A., Wilson, A. S., Arnaud, K. A., Terashima, Y., \& Young, A. J. 2002, ApJ, 565, 195

Stanford, S. A., Holden, B., Rosati, P., et al. 2001, ApJ, 552, 504

Stevens, J. A., Ivison, R. J., Dunlop, J. S., et al. 2003, Nature, 425, 264

Taylor, G. B., Barton, E. J., \& Ge, J. 1994, AJ, 107, 1942

Tozzi, P., Rosati, P., Nonino, M., et al. 2001, ApJ, 562, 42

van Ojik, R., Roettgering, H. J. A., Miley, G. K., \& Hunstead, R. W. 1997, A\&A, 317, 358

Venemans, B. P., Kurk, J. D., Miley, G. K., et al. 2002, ApJ, 569, L11

Wilson, A. S., Young, A. J., \& Shopbell, P. L. 2000, ApJ, 544, L27

Windhorst, R. A., Keel, W. C., \& Pascarelle, S. M. 1998, ApJ, 494, L27

Young, A. J., Wilson, A. S., Terashima, Y., Arnaud, K. A., \& Smith, D. A. 2002, ApJ, 564, 176

Yuan, W., Fabian, A. C., Celotti, A., \& Jonker, P. G. 2003, MNRAS, 346, L7 